\title{
Hematopoietic Stem Cells Count and Remember Self-Renewal Divisions
}

Jeffrey M. Bernitz ${ }^{1,2,3}$, Heun-Suk Kim¹,2,3, Ben MacArthur ${ }^{4}$, Hans Sieburg ${ }^{5}$, Kateri Moore $e^{1,2}$

${ }^{1}$ Department of Developmental and Regenerative Biology; ${ }^{2}$ Black Family Stem Cell Institute; ${ }^{3}$ The Graduate School of Biomedical Sciences; Icahn School of Medicine at Mount Sinai, One Gustave L. Levy Place, Box 1496, New York, NY 10029, USA. ${ }^{4}$ Mathematical Sciences and Centre for Human Development, Stem Cells and Regeneration, Faculty of Medicine, University of Southampton, Southampton SO17 1BJ, UK Institute for Life Sciences, University of Southampton, Southampton SO17 1BJ, UK. ${ }^{5}$ Vaccine Research Institute of San Diego, San Diego, CA 92121, USA

\section{Summary}

The ability of cells to count and remember their divisions could underlie many alterations that occur during development, aging, and disease. Here, we tracked the cumulative divisional history of slow-cycling hematopoietic stem cells (HSCs) throughout adult life. Doing so revealed a rare fraction of minimally divided HSCs that contained all the long-term HSC (LT-HSC) activity within the aging HSC compartment. During adult life this population asynchronously completes four symmetric self-renewal divisions, expanding its size before entering a state of permanent dormancy after completing the fourth division. We demonstrate that this expansion only occurs if the time between cell divisions extends 
progressively until permanent dormancy is achieved after the fourth division. Our model also shows that age-related phenotypic changes within the HSC compartment are divisional history dependent. These results reveal that HSCs count and remember their divisional history and provide evidence that these phenomena may underlie HSC aging.

\section{Highlights}

- A rare population of dormant LR-HSCs persists throughout adult life.

- Only $1 \%$ of repopulating cells within the aging HSC compartment are LTHSCs.

- LR-HSCs divide symmetrically 4 times throughout adult life to accumulate with age.

- HSCs count and retain a memory of their cell division events in vivo.

\section{eTOC Blurb}

Tracking retention of Histone 2B green fluorescent protein (H2BGFP) label throughout adult life reveals a small population of dormant hematopoietic stem cells (HSCs) that contain all long-term stem cell activity in aging bone marrow. Using H2BGFP to track cell divisions over time showed that the dormant HSC population undergoes four symmetric self-renewal events that expand the stem cell pool with age. Upon a fifth cell division, long-term regenerative potential is lost revealing HSCs count their cell divisions in vivo.

\section{Introduction:}


Under certain circumstances cells can adapt or modify their behaviors in response to previous events. This "cellular memory" is known to play crucial roles in organismal homeostasis and normal physiology. Yeast that exhibit strong responses to DNA damage demonstrate growth defects heritable for many generations not observed by weak responders (Burrill and Silver, 2011). Activated synapses of neurons in Drosophila and Aplysia convert CPEB to a selfpropagating prion-like state, allowing specific synapses to "remember" their previous activation and facilitate long-term memory storage (Majumdar et al., 2012; Si et al., 2003a; Si et al., 2003b). Additionally, during development the differentiation of cells from a pluripotent to a committed state is generally considered an irreversible process that must be written to memory to prevent aberrant expression of alternative cell type-specific genes (Ang et al., 2011; Efroni et al., 2008). Another manifestation of cellular memory could be through the counting of cell divisions. Indeed, such a mechanism has been proposed to account for the loss of function and senescence of cells with age, where cells "count" cell divisions via telomere length (Harley et al., 1990). While cells may be storing information about their cumulative divisional history, technical challenges associated with accurately documenting large numbers of cell divisions in vivo have largely precluded observations of these phenomena.

HSCs are bone marrow resident stem cells responsible for maintaining the hematopoietic system throughout life, and largely reside in a quiescent state (Nakamura-Ishizu et al., 2014). Studies on HSC cycling kinetics demonstrate the presence of a rare population of dormant HSCs that divide minimally over time 
and retain all the serially transplantable hematopoietic regenerative potential in the bone marrow (BM) (Foudi et al., 2009; Qiu et al., 2014; Wilson et al., 2008). These dormant HSCs are identified by their ability to retain a pulsed histone 2Bgreen fluorescent protein (H2BGFP) label, and are also referred to as labelretaining HSCs (LR-HSCs). LR-HSCs are believed to act as a reserve stem cell population as they are resistant to chemotherapeutic agents but efficiently activate in response to stress to replenish lost hematopoietic populations (Essers et al., 2009; Wilson et al., 2008). However, while LR-HSCs are known to retain labels for close to a year (van der Wath et al., 2009; Wilson et al., 2008), their role, or presence, throughout the entirety of adult life remains unexplored.

Upon aging HSC regenerative potential declines. Aged HSCs demonstrate reduced self-renewal (Dykstra et al., 2011), impaired homing and engraftment upon transplantation (Dykstra et al., 2011; Morrison et al., 1996), and myeloidbiased differentiation (Benz et al., 2012; Cho et al., 2008; Muller-Sieburg et al., 2004; Rossi et al., 2005; Sudo et al., 2000). Paradoxically, HSCs are also reported to increase in number with age, both in mice and humans (Beerman et al., 2010; Cho et al., 2008; Dykstra et al., 2011; Morrison et al., 1996; Pang et al., 2011; Sudo et al., 2000). Current models of HSC aging suggest that HSC numbers expand in order to compensate for defects that naturally occur with age, but evidence for this hypothesis is lacking (Geiger et al., 2013).

Our previous work demonstrated that as HSCs progressively divide over time, they gradually lose regenerative and self-renewal capacity (Qiu et al., 2014). Additionally, many of the characteristics of aged hematopoiesis can be 
replicated by enforcing HSC proliferation (Beerman et al., 2013). Thus we hypothesized that HSC aging may be a direct result of extensive proliferative history accrued throughout life, but there remains a need to understand how HSCs naturally cycle over time and into an aged system.

Here we report a rare population of HSCs capable of retaining H2BGFP for at least 22 months in vivo. This population of stable label-retaining HSCs (sLR-HSCs, cells capable of retaining a pulsed H2BGFP label for greater than 10 months) contains all cells capable of robust multi-lineage engraftment in primary and secondary transplantation (hereafter referred to as long-term (LT-) HSCs) in aging BM when assayed at the clonal level. We find that LR-HSCs reduce in frequency within the stem cell compartment but accumulate in absolute number in the BM with age. A simple mathematical analysis shows that this accumulation is well-described by a model in which LR-HSCs undergo symmetric self-renewal events that rapidly extend the time until the next cell cycle initiation with each successive cell division, until LR-HSCs reach a state of complete dormancy after four self-renewal events. Our studies demonstrate a direct link between single cell division events and HSC behavior, and suggest that HSCs are capable of counting and retaining a memory of their cell division events during adult life.

\section{Results:}

\section{A small population of LR-HSCs exists throughout adult life}

To assess the cumulative divisional history of HSCs throughout adult life, we used a tet-off hematopoietic stem and progenitor cell (HSPC) specific H2BGFP label-retaining system (Qiu et al., 2014). This system allows HPSCs to 
be continuously labeled with H2BGFP throughout development and ontogeny, ensuring thorough and robust labeling of the HSC compartment (cells defined as Lineage $/$ Sca- $1^{+} / \mathrm{CKit}^{+} / \mathrm{CD} 48^{-} / \mathrm{Flk} 2^{-} / \mathrm{CD} 150^{+}$) at the onset of doxycycline (dox) chase (Figure 1B and S1A). We placed young adult mice (7-18 weeks old) on extended dox chases ranging from 3-22 months, allowing us to assess the cumulative divisional history of the HSC compartment at various time points throughout life (Figure 1A). Analyzing the kinetics of H2BGFP loss, we find that the percentage of $\mathrm{H}_{2} \mathrm{BGFP}{ }^{+}$cells rapidly declines initially, but eventually plateaus after 10 months of dox chase and remains relatively constant for chases lasting nearly 2 years (Figure 1C). After 10-22 months on dox, sLR-HSCs represent 3.24 $\pm 1.26 \%$ (mean $\pm \mathrm{SD}$ ) of the HSC compartment (Figure 1D).

To eliminate the possibility that sLR-HSCs are an artifact of leaky H2BGFP expression in our system, we analyzed the HSC compartment of mice raised on dox via the pregnant mother throughout development and maintained on dox until adulthood (Figure S1B). In mice born and raised on dox treatment for up to a full year, we found no H2BGFP expression above background levels (Figure S1E and S1F), indicating that H2BGFP is not inappropriately expressed in the presence of dox, and that our population of SLR-HSCs is not due to leaky H2BGFP expression. Together these results demonstrate a small population of cells within the HSCs compartment divisional minimally throughout the majority of adult life.

\section{sLR-HSCs contain all LT-HSC activity within aging BM}


To test the function of sLR-HSCs, we performed competitive transplantations on aging GFP ${ }^{\mathrm{LO}}, \mathrm{GFP}^{\mathrm{Hi}}$ (sLR-HSCs), and Total HSC populations sorted from 19 month-old mice chased with dox for 15 months (Figure 1E). In primary recipients, all three populations stably engrafted, but the GFP ${ }^{\mathrm{Hi}}$ population demonstrated significantly higher levels of chimerism compared to the GFP ${ }^{\mathrm{LO}}$ and Total populations (Figure 1F and 1G). Importantly, the Total HSC population demonstrated intermediate levels of chimerism in primary recipients that fell between the GFP ${ }^{\mathrm{Hi}}$ and GFP ${ }^{\mathrm{Lo}}$ repopulation curves (Figure $1 \mathrm{~F}$ and $1 \mathrm{G}$ ). In secondary hosts, only the Total and GFP ${ }^{H i}$ populations demonstrated sustained secondary engraftment, while engraftment by GFP ${ }^{\mathrm{LO}} \mathrm{HSCs}$ progressively declined until exhaustion by the end of 24 weeks (Figure $1 \mathrm{~F}$ and 1G, and Table S1). Interestingly, in secondary transplants the chimerism of Total and GFP ${ }^{H i}$ cells were indistinguishable, suggesting that self-renewal capacity and secondary engraftment potential are manifested only by SLR-HSCs within the aging HSC compartment.

At sacrifice we analyzed regeneration of primitive HSPC compartments in the BM (Figure $1 \mathrm{H}$ ). We found that after both transplants, GFP ${ }^{\mathrm{Hi}}$ cells generated higher HSPC chimerism compared to GFP ${ }^{L \circ}$ cells (Figure $1 \mathrm{l}$ and $1 \mathrm{~J}$ ). However, when compared to the Total population, GFP ${ }^{\mathrm{Hi}}$ cells showed greater regeneration only in the HSC population after primary transplant, with no difference after secondary transplant. Overall, these results are consistent with the peripheral blood reconstitution, whereby the small proportion of GFP ${ }^{\mathrm{Hi}}$ cells within the Total HSC compartment must be responsible for the levels of HSPC regeneration in 
primary and secondary recipients. Taken together the data show that the small

proportion of GFP ${ }^{H i}$ sLR-HSCs_identifiable only by their minimal divisional history—contain all of the LT-HSC potential within the aging HSC compartment.

\section{Increased divisional history marks increased myeloid potential}

Aged HSCs demonstrate increased myeloid cell output upon transplantation (Beerman et al., 2010; Benz et al., 2012; Cho et al., 2008; Dykstra et al., 2011; Gekas and Graf, 2013; Sudo et al., 2000). To understand how proliferative history correlates with lineage reconstitution we measured lineage output in the blood of primary and secondary transplant recipients. While all three aging HSC populations show increased myeloid output in primary transplant, similar to the blood of unmanipulated aging mice (Figure 1K), only GFP $^{\mathrm{Hi}}$ cells maintained this myeloid output in secondary recipients (Figure 1L). As GFP ${ }^{L o}$ grafts exhausted by the end of secondary transplantation (Figure $1 \mathrm{~F}$ and Table S1), we hypothesized that this fraction may be enriched for myeloid progenitors lacking self-renewal capacity found within the HSC compartment.

To examine the myeloid potential of the HSC compartment we examined CD41 expression. While highly enriched for bona-fide HSCs, the HSC compartment is heterogeneous. Within the HSC compartment CD41 has been reported to mark myeloid and megakaryocyte progenitors (Haas et al., 2015; Yamamoto et al., 2013), and it's expression increases with age (Gekas and Graf, 2013). Given that myeloid-restricted repopulating cells accumulate in aged mice (Dykstra et al., 2011; Sudo et al., 2000), we hypothesized that by examining 
CD41 expression we would better understand both the heterogeneity and the myeloid potential within aging HSC compartments.

We first compared the primitive stem and progenitor compartments of young and aging mice. Consistent with other reports (Beerman et al., 2010; Dykstra et al., 2011), we find the proportion, frequency, and absolute number of $\mathrm{CD} 150^{+}$cells marking the HSC compartment increase with age (Figure 2A, B, $\mathrm{S} 2 \mathrm{~A}$, and S2B). However, the vast majority of this expanded compartment is $\mathrm{CD} 1^{+}$(Figure 2C, 2D, S2C, and S2D), indicative of the phenotype of myeloid progenitors (Yamamoto et al., 2013). Within the HSC compartment CD41 ${ }^{+}$cells also accumulate with age (Figure 2E, 2F, S2E and S2F), consistent with previous findings (Gekas and Graf, 2013). Dissecting the HSC compartment further using label-retention, we find the Total and GFP ${ }^{\text {Lo }}$ populations show similar increases in $\mathrm{CD} 41$ expression with age, but young and aging GFP ${ }^{\mathrm{Hi}}$ populations are depleted of $\mathrm{CD} 41^{+}$cells (Figure 2E and 2G). Snapshot cell cycle analysis demonstrates $C D 41^{+}$cells have a lower percentage of cells in $\mathrm{G}_{0}$ than $\mathrm{CD} 41^{-}$ cells (Figure S2I and S2J), and retain lower levels of H2BGFP, suggesting that within the CD41 ${ }^{+}$cells $\mathrm{HSC}$ compartment cycle more frequently than $\mathrm{CD} 41^{-}$cells (Figure S2K and S2L). These data demonstrate that CD41 expression on HSCs correlates with diminished quiescence and a higher rate of cycling in vivo, which may in part explain how $\mathrm{CD} 41^{+}$cells within the total HSC compartment accumulate with age.

To examine megakaryocyte potential, we performed single cell megakaryocyte colony forming unit assays (Figure S3). We found that the Total, 
GFP ${ }^{\mathrm{Lo}}$, and $\mathrm{GFP}^{\mathrm{Hi}} \mathrm{HSC}$ populations from young and aging animals all generated colonies that contained large round cells with megakaryocte morphology (Figure S3E and S3G), but only the Total and GFP ${ }^{\mathrm{LO}}$ populations generated colonies exclusively containing large round megakaryocytes (Figure S3D and S3G). Taken together with the increase of CD41 expression in the Total and GFP ${ }^{\mathrm{LO}}$ HSC compartments, and the increased myeloid reconstitution after primary but not secondary transplant suggests that the aging HSC compartment may be dominated by myeloid progenitors, rather than functional HSCs.

\section{Clonal analysis of aging HSC populations based on label-retention and CD41 expression.}

Lineage reconstitution, cell-surface marker, and cell cycle analyses suggested that the expansion of the HSC compartment with age may be due to expansion of myeloid progenitors rather than expansion of bona-fide HSCs. To test this hypothesis, we performed limiting cell number serial transplants of aging HSCs populations based on label-retention and CD41 expression. We sorted HSCs from 19-month old mice chased with dox for 17 months into four populations: GFP ${ }^{\mathrm{Hi}} \mathrm{CD} 41^{-}, \mathrm{GFP}^{\mathrm{Hi}} \mathrm{CD} 41^{+}, \mathrm{GFP}^{\mathrm{Lo}} \mathrm{CD} 41^{-}$, and $\mathrm{GFP}^{\mathrm{Lo}} \mathrm{CD} 41^{+}$(as in Figure 2E). 15 cells from each population were then competitively transplanted and the donor-derived myeloid, B cell, and T cell contributions to peripheral blood were followed for 24 weeks in primary and secondary hosts. Because we were looking for any form of repopulating activity and not just stem cell activity, we considered a mouse repopulated if at any point during primary transplantation we found any donor-derived lineage to contribute to $>0.1 \%$ of total circulating 
peripheral blood leukocytes (Figure 4A; (Yamamoto et al., 2013)). Based on this definition of repopulation, we performed limiting dilution analysis to determine the clonality of our transplanted populations, revealing that most of the mice were repopulated at or very near clonal levels (Table S2).

As a further measure to validate clonality, we transplanted BM from a single primary host into two secondary hosts. Daughter HSCs generated from the same initial parent HSC in vivo show synchronous repopulation kinetics when transplanted into separate hosts (Muller-Sieburg et al., 2002). Thus, in secondary transplants we followed the kinetics of total donor-type $\left(\mathrm{CD} 45.2^{+}\right)$repopulation in paired secondary hosts and measured the degree of synchronicity between them using Hamming distance clustering (Sieburg and Muller-Sieburg, 2004). 37 of the 46 mice initially transplanted survived through 6 months in primary hosts. 24 of those demonstrated repopulation above threshold in primary recipients and were used for paired secondary transplantation. 23 of the 24 secondary transplant pairs were used for Hamming distance analysis, as one of the mice in the $24^{\text {th }}$ pair died before the first peripheral blood analysis. From the 23 pairs analyzed, 18 demonstrated synchronous kinetics of donor-type repopulation in secondary hosts, indicative of clonal repopulation (Figure S3). These analyses allowed us to conclude that most of the mice were repopulated clonally, with a few exceptions that demonstrated oligoclonal repopulation.

For analysis of repopulating patterns, all 24 repopulated mice were considered. We followed donor-derived contribution to $\mathrm{B}, \mathrm{T}$, and myeloid cells from each sorted population over the course of 24 weeks in both primary and 
secondary transplantations (Figure 3A). From the 24 repopulated mice, we observed 5 distinct repopulation patterns: myeloid-restricted, which only repopulated myeloid cells; bipotent progenitor, which repopulated the myeloid and B cell lineages; short-term HSC (ST-HSC), which showed transient repopulation of all three lineages, with donor chimerism of at least one lineage dropping below threshold prior to the end of the primary transplantation; intermediate-term HSC (IT-HSC), which repopulated all three lineages, but had at least one lineage drop below threshold prior to the end of secondary transplantation; and LT-HSC patterns, which maintained robust repopulation in all three lineages analyzed through both primary and secondary transplantation (Figure 3B). Based on these definitions, we quantified the heterogeneity of repopulation patterns within each sorted population. Consistent with our polyclonal transplantation data (Figure 1), LT-HSC repopulation was exclusively confined to the GFP ${ }^{H i}$ cell fractions (Figure $3 \mathrm{C}$ ). GFP ${ }^{\mathrm{Hi}}$ cells demonstrated only IT- and LT-HSC repopulation patterns, which were similarly represented regardless of CD41 expression (Figure $3 C$ ). In contrast, the GFP ${ }^{L O}$ cells demonstrated limited self-renewal potential and greater heterogeneity of repopulating patterns. Additionally, we found CD41 expression enriched for myeloid-restricted repopulating cells within the GFP ${ }^{\text {Lo }}$ compartment (Figure 3C). Finally, we used the proportion of repopulating cell types found within each sorted HSC fraction to extrapolate the representation of repopulating cell types found within the total aging HSC compartment. Remarkably, LT-HSCs only represented roughly $1 \%$ of the repopulating cells of the Total aging HSC 
compartment (Figure 3C). Instead, we found that cells with limited self-renewal (myeloid progenitors, bipotent progenitors, and ST-HSCs) represent $\sim 80 \%$ of repopulating cells of the aging HSC compartment, with $38 \%$ of repopulating cells having a myeloid progenitor identity (Figure 3C).

After primary transplant, we analyzed recipient BM for donor-derived regeneration of various stem and progenitor cell compartments (Figure 3D). When we compared regeneration efficiencies based on retrospectively assigned repopulating cell type, as expected the most primitive cell types (LT- and ITHSCs) show the most robust regeneration efficiencies, while the more developmentally restricted cell types (ST-HSC, Bipotent, and Myeloid progenitors) showed reduced regeneration capacities. Interestingly, both LT- and IT-HSC patterns showed impaired regeneration of common lymphoid progenitors (CLPs), consistent with aging HSC phenotypes (Rossi et al., 2005). When comparing regeneration based on sorted cell phenotype, GFP ${ }^{H i}$ cells repopulated each compartment with higher efficiency than GFP ${ }^{\text {Lo }}$ cells. Notably, both GFP ${ }^{\mathrm{Hi}} \mathrm{CD} 41^{+}$and $\mathrm{GFP}{ }^{\mathrm{Hi}} \mathrm{CD} 41^{-}$cells generated $\mathrm{CD} 41^{-}$and $\mathrm{CD} 41^{+} \mathrm{HSC}$ with similar efficiencies, suggesting that while CD41- HSCs may reside at the apex of the hematopoietic hierarchy in young mice, with age this hierarchical organization is dysregulated in a transplantation setting. Within the GFP ${ }^{\mathrm{Lo}}$ fractions, GFP ${ }^{\mathrm{LO}} \mathrm{CD} 41^{-}$cells were more efficient at regenerating the more primitive compartments than the GFP ${ }^{\mathrm{Lo}} \mathrm{CD} 41^{+}$cells, which primarily made myeloid progenitor fractions (Figure 3D). 
Together, these data support the conclusion that LT-HSCs residing in aging BM are exclusively found in the rare population of SLR-HSCs ( 3\%), and that the vast majority of the HSC compartment ( $97 \%)$ consists of repopulating cells with limited self-renewal and restricted differentiation potential. Additionally, these results provide evidence that LT-HSCs can be completely separated from contaminating progenitor populations by the use of H2BGFP label-retention within the aging HSC compartment. This ability allows us to see that the dramatic expansion of the phenotypic HSC compartment with age is primarily due to the expansion of proliferative progenitors with limited regenerative potential.

\section{Tracking GFP label dilution reveals LR-HSCs symmetrically self-renew throughout adult life.}

Next, we wanted to address how functional HSC numbers expand with age. While the HSC compartment expands dramatically with age (Beerman et al., 2010; Dykstra et al., 2011; Rossi et al., 2005; Sudo et al., 2000), functional LTHSC numbers within aging BM expand but to a lesser extent (Cho et al., 2008; Morrison et al., 1996; Sudo et al., 2000). Our previous studies demonstrated, and this study confirms at the clonal level, that independent of cell surface marker expression LR-HSCs are LT-HSCs (Figure 4; (Qiu et al., 2014)). Thus we investigated the phenomena of functional HSC expansion further by utilizing information from our total LR-HSC populations, regardless of CD41 expression.

First we examined H2BGFP label retention in young mice. Upon specific examination of the GFP ${ }^{\mathrm{Hi}} \mathrm{HSC}$ fraction in young mice, we find 5 distinct 
subpopulations of cells associated with GFP peaks (Figure 4A). These peaks are identifiable using the proliferation index utility in Flowjo software (Treestar), indicating that they are associated with successive cell divisions where divisional history increases as cells progress from peak 0 to peak 4 (Figure 4A). To test if the observed peaks represent actual cell division events we extracted GFP intensity data for 1568 single GFP ${ }^{\mathrm{Hi}} \mathrm{HSC}$ s from young mice and analyzed the relative positions of each of the observed GFP peaks. We found that the positions of these peaks are very well described by a simple model in which GFP dilutes by a factor of 2 through each cell division (Figure 4C). This suggests that these peaks are indeed marking cell division events, and that we can use the GFP peaks found within the GFP ${ }^{H i}$ cells to quantify cell divisions over time in vivo.

Next we compared the GFP peaks from young and aging LR-HSC populations. Although LR-HSCs from young mice display 5 distinct GFP peaks, $97.4 \%$ of sLR-HSCs from aging mice are found within peak 4 (Figure $4 \mathrm{~A}$ and $4 \mathrm{~B}$ ). Given that we can use the GFP peaks to quantify divisional history, this suggests that the vast majority of sLR-HSCs have completed 4 cell divisions. We then quantified the absolute number of LR-HSCs in the leg bones of young and aging mice. Remarkably, even though LR-HSC representation decreases between young and aging mice (Figure 4D), we find that the absolute number of LR-HSCs within the BM increases with age (Figure 4E).

To summarize, our transplantation experiments indicate that all LT-HSC activity is contained within the LR-HSC fractions in both young (Qiu et al., 2014) 
and aging mice (Figures 1 and 3 ). Thus, in combination, our findings show that 1 ) functional capacity of LR-HSCs does not alter with age, 2) the absolute number of LR-HSCs increases over time, 3) the majority of sLR-HSCs have divided 4 times during the span of dox chase, and 4) H2BGFP is not re-expressed in the presence of dox. Taken together these data suggest that expansion of LR-HSC numbers must be due to symmetric self-renewal events of LR-HSCs during the lifespan of the mouse.

To experimentally test this, we used a simple model to estimate the expansion capacity of LR-HSCs from young mice (Figure 4F). First we quantified the absolute numbers of LR-HSCs found in each peak in young mice. As $>95 \%$ of sLR-HSCs in aging mice are found in peak 4, we modeled LR-HSC expansion with age as population doublings of each GFP peak as the cells divide to dilute their H2BGFP level from their starting peak in young mice to peak 4. This generated a prediction of SLR-HSC numbers once every young LR-HSC had divided enough to match the GFP intensity found in peak 4 . We found that the predicted values $\left(799_{ \pm} 491\right.$ cells/bone) closely matched the mean and variance of the experimentally acquired data from aging mice $(707 \pm 316$ cells/bone; Figure 4D). Of note, this model assumes no loss of LR-HSC number due to cell death, differentiation, or cell division events resulting in GFP dilution to levels below peak 4 . The correlation between predicted and experimental data suggests LRHSCs cycle symmetrically, generating two daughter LR-HSCs with each division, a maximum of 4 times, and that LR-HSCs found in peak 4 in young mice remain 
deeply dormant throughout adult life without losing considerable cell numbers due to contribution to active hematopoiesis.

\section{LR-HSC cell cycle times extend rapidly with divisional history until permanent dormancy is reached after 4 cell divisions.}

To investigate this further, we used LR-HSC numbers from young mice as initial conditions for a series of mathematical models to better determine whether divisional history impacts LR-HSC cell cycle progression (see Materials and Methods). The first, and simplest, model assumes that cells divide at a constant rate regardless of divisional history (Figure 4G; Constant). Because GFP will ultimately dilute away in all cells under this model, it cannot explain LR-HSC accumulation with age and suggests that divisional history affects LR-HSC cell cycle progression time. To account for this accumulation, we considered a revised model in which individual cells cycle at a constant rate until they have divided 4 times, at which point they stop dividing. This modified model explains the data well (Figure 4G, Step Function), but makes the strong assumption that cells cycle normally until they have divided 4 times and then suddenly stop dividing. We reasoned that this was likely not the case, but rather that successive cell divisions become progressively less likely to occur the more a cell has divided previously. This prompted us to further adjust our model in order to better understand how cell cycle entry alters with divisional history. Both linear and exponential extensions of cell cycle entry times with divisional history did not accurately predict cell numbers in aging mice. However, a super-exponential extension was found to fit the experimental data well (Figure 4G). In this model, 
predicted cell cycle times for cells in peak 0,1 , and 2 are short, but as cells undergo further divisions, their cycle times rapidly increase until the expected cell cycle time for cells in peak 4 is 1256 days, or roughly 3.5 years (i.e. permanently dormant; Figure 4G, red curve). Importantly, this model also predicts rather than assumes that cells effectively reach a permanently dormant state after 4 cell divisions, and therefore explains how cells found in peak 4 in young mice are still present in aged mice.

To test these predictions, we examined the change in GFP peak distribution and LR-HSC absolute numbers after various lengths of dox chase. Consistent with the super-exponential model of cell cycle extension, we find cells in peaks 0,1 , and 2 are quickly lost, with few of these cells remaining after a 3-4 chase, and the vast majority of LR-HSCs are found in peak 4 as soon as 9 months after the start of dox (Figure 4H). Additionally, we find that LR-HSC doubling is seen as early as 12 months after dox treatment and is maintained until at least 22 months on dox, corresponding to 25 months of age (Figure $4 \mathrm{I})$. If peak 4 cells were to continue to proliferate during these long chases, LR-HSC numbers should decrease over time. Rather we find that LR-HSCs numbers are stable, suggesting that indeed once cells reach peak 4 they are permanently dormant (Figure 4I). We found one exception, where a 24 month-old mouse had significantly lower numbers of SLR-HSCs than what would be predicted (Figure $4 \mathrm{H}$, red asterisk). Upon dissection, this mouse had an enlarged liver with multiple tumors (data not shown), indicative of a severe systemic stress, which may have led to the activation and depletion of dormant SLR-HSCs in this mouse. 
Additionally, the cell cycle profile between young and aging peak 4 cells showed greater than $90 \%$ of cells in $\mathrm{G}_{0}$ suggesting that the cell cycle of these cells do not change with time (Figure $4 \mathrm{~J}$ and S5A). Taken together, these analyses suggest that LR-HSCs adopt a permanently dormant state after 4 symmetric self-renewal divisions, which double the LR-HSC compartment with time, demonstrating that LR-HSCs count their cell divisions throughout life.

\section{$\mathrm{CD} 1^{+}$sLR-HSCs are generated from CD41- ${ }^{-}$RR-HSCs throughout adult life}

In order to see if a cell division counting mechanism could underlie other changes seen in the HSC compartment with age, we re-examined the hierarchical relationship between $\mathrm{CD} 41^{-}$and $\mathrm{CD} 41^{+} \mathrm{LR}$-HSCs. CD $41^{+}$cells that function with LT-HSC potential have not been described at the clonal level in young adult mice (Yamamoto et al., 2013), but in the aging HSC compartment CD $41^{+}$HSCs have been shown to reside at the top of the hematopoietic hierarchy (Gekas and Graf, 2013). In order to understand how $\mathrm{CD} 41^{+}$cells develop LT-HSC potential with aging (Figure 3C), we compared the H2BGFP label dilution of $\mathrm{CD} 41^{-}$and $\mathrm{CD} 41^{+}$cells within the GFP ${ }^{\mathrm{Hi}} \mathrm{LR}-\mathrm{HSC}$ fractions of young and aging mice. Comparing the GFP peak distributions of $\mathrm{CD} 41^{-}$and CD41 ${ }^{+}$LR-HSCs, we observe that young CD41- LR-HSCs are distributed throughout all five GFP peaks, while the vast majority of young $\mathrm{CD} 41^{+}$cells are primarily found in peak 4' similar distribution to the aging compartment (Figure S5B). CD $41^{+}$LR-HSCs increase considerably in number and frequency with age (Figure S5E, S2G, and S2H), but given that the majority of CD41 $1^{+} \mathrm{LR}-\mathrm{HSC}$ can no longer divide and retain LT-HSC potency, it would not be possible for young 
CD $41^{+}$LR-HSC to generate the expanded population of CD41 $1^{+}$SLR-HSCs found in aging mice. Thus, we wondered whether CD41- LR-HSCs from young mice are responsible for generating the expanded population of $\mathrm{CD} 41^{+} \mathrm{sLR}-\mathrm{HSCs}$ found in aging mice.

Direct comparison of $\mathrm{CD} 41^{-}$and $\mathrm{CD} 41^{+} \mathrm{LR}-\mathrm{HSC}$ s from young mice showed that CD41- LR-HSCs have a greater proportion of cells in with a minimal divisional history in GFP peaks 0-3 than CD41 $1^{+}$LR-HSC (Figure S5C). However, with aging both $\mathrm{CD} 41^{-}$and $\mathrm{CD} 41^{+}$sLR-HSCs have similar GFP distributions where most cells have completed 4 cell divisions (Figure S5C). To quantify this we measured GFP fluorescence intensities of each population and observed brighter GFP in young CD41- over CD41 $1^{+}$LR-HSCs, but find the intensities are indistinguishable in aging mice (Figure S5D). Importantly, CD41 ${ }^{-}$cell GFP intensity drops with age, but the GFP intensity of the CD $41^{+}$population does not change. As $\mathrm{CD} 41^{+}$cells more rapidly proliferate than $\mathrm{CD} 41^{-}$cells (Figure S2J) and the CD $41^{+} \mathrm{LR}-\mathrm{HSC}$ population expands with time (Figure S5E), either CD41LR-HSCs found in GFP peaks 0-3 directly generate CD41 $1^{+} \mathrm{LR}-\mathrm{HSCs}$, or that CD41 expression becomes dynamic with age and CD41- LR-HSCs turn on CD41 expression in a manner that does not alter their regenerative potential (Figure 3C and 3D).

To test this hypothesis, we compared the change in absolute numbers of CD41 $1^{-}$and $\mathrm{CD} 41^{+} \mathrm{LR}-\mathrm{HSC}$ s with age to their predicted expansion capacity from young mice (Figure 4F). We found that while CD41- LR-HSCs expand minimally from young to aging mice, young CD41- LR-HSCs have the capacity to expand 
nearly 3 fold and still retain the GFP label (Figure S5E). In contrast, CD $41^{+}$LRHSCs demonstrate a 3 fold expansion from young to aging mice, but contain no endogenous expansion capacity (Figure S5E). Thus in order for CD41 $1^{+} \mathrm{LR}-\mathrm{HSC}$ numbers to expand, CD41- LR-HSCs must generate CD41 ${ }^{+}$LR-HSCs as they divide. To precisely gauge CD41 ${ }^{-}$contribution to the CD $41^{+} \mathrm{LR}-\mathrm{HSC}$ pool over time, we modified our super exponential model of cell cycle extension to allow CD41- LR-HSCs to gain CD41 expression with a fixed probability each time they divide. This model accurately fits the data for both CD $41^{-}$and CD $41^{+} \mathrm{LR}-\mathrm{HSC}$ expansion in aging mice, and estimates that in order for the CD41 $1^{+} \mathrm{LR}-\mathrm{HSC}$ pool to expand, approximately 1 in 10 division events within the CD41- LR-HSC pool must give rise to a CD41 $1^{+}$LR-HSC (Figure S5F). This demonstrates that in the context of homeostatic hematopoiesis, CD41- LR-HSCs reside at the apex of the hematopoietic hierarchy, generating the expanded CD $41^{+}$LR-HSC population found with age.

\section{Discussion}

Cells take in and store information about previous events in their history that dictates how they will subsequently behave. Examples range from responding to differentiation and specification cues during development, to the induction of $V(D) J$ recombination in lymphoctyes after encountering a specific pathogen (Burrill and Silver, 2010). Here we utilized H2BGFP label retention to investigate how HSC cell divisions accrued naturally through the process of aging impact on their regenerative potential. 


\section{Divisional history and heterogeneity of the aging HSC compartment}

Previous work using H2BGFP label-retention to characterize HSCs revealed that the fewer times HSCs cycle over time, the greater their regenerative potential (Foudi et al., 2009; Qiu et al., 2014; Wilson et al., 2008). Within the functionally heterogeneous LSK compartment, we were able to show that label retaining cells could long term repopulate a mouse at a frequency of 1 in 2.9 cells (Qiu et al., 2014). Here, we furthered on these studies by using label-retention to dissect heterogeneity within the aging HSC compartment. We find that on the clonal level sLR-HSCs function exclusively with IT- or LT-HSC potential, but also proliferative non-LR-HSCs, which represent the vast majority of the aging HSC compartment, contain a diverse class of progenitors with reduced self-renewal and differentiation potential that do not function with HSC potential. This non-LR-HSC population may be the source of myeloid-repopulating cells described in clonal transplantation experiments (Dykstra et al., 2011; Sudo et al., 2000; Yamamoto et al., 2013), and supports the conclusion that myeloid-repopulating cells are generated as a function of accumulated divisional history (Dykstra et al., 2011). The degree of functional heterogeneity within the non-LR-HSC population, and indeed the HSC compartment on the whole, supports increased evidence that cell surface markers alone cannot select for a functionally homogeneous populations when analyzed at the clonal level (Dykstra et al., 2011; Paul et al., 2015; Perie et al., 2015; Yamamoto et al., 2013). Additionally, the reduced regenerative capacity of the proliferative non-LR-HSC population is also 
consistent with studies demonstrating that increasing cell cycle often reduces HSC function (Bowie et al., 2006; Pietras et al., 2011).

It is worth noting that our sLR-HSCs do not repopulate with absolute efficiency. This could be explained by a number of defects that characterize aged HSC populations, including defects in cell cycle entry, replication stress, and repair of DNA damage accumulated from long-term quiescence (Beerman et al., 2014; Flach et al., 2014; Mohrin et al., 2010; Rossi et al., 2007). However, all cells that do repopulate from transplanted sLR-HSCs do so with LT- or IT-HSC functional potential, and many of the cells that we categorize as IT-HSCs would be defined as LT-HSCs by less-stringent definitions. Thus, we suggest that labelretention is a powerful tool that can be used to discriminate cells with HSC potential from populations of functionally heterogeneous progenitors, and especially in the context of and aged system. This would prove beneficial when studying defects of aging HSCs by eliminating confounding results that describe defects among the diverse HSPCs invariably contained within the HSC compartment.

\section{Tracking HSC divisional history throughout adult life reveals HSCs document their cell divisions}

The use of a viable label-retaining system enabled us to track and quantify cell divisions over the lifespan of a mouse. We find that LT-HSCs are capable of undergoing four cell divisions prior to the loss of LT-HSC potential, which we can visualize with the dilution of H2BGFP fluorescence intensity over time. This 
experimentally supports the mathematical models of previous work on LR-HSCs, which predicted that HSCs divide five times during the lifespan of a mouse (Wilson et al., 2008). When considered together with our data, this fifth cell division would result with a complete loss of LT-HSC potential, and most likely the initiation of an irreversible decline of the hematopoietic system.

Our approach also enabled us to make inferences about LR-HSC expansion and cell division patterns. Our approach allowed us to conclude that age associated expansion can be reached only if 1) LR-HSCs exclusively undergo symmetric self-renewal divisions throughout life (i.e. they generate two daughter LR-HSCs with every cell division), and 2) that they reach permanent dormancy after completing their fourth cell division. The LR-HSC compartment divides asynchronously (Figure 4B), but regardless of when a cell completes it's fourth division from the onset of dox chase we can assume it enters a permanently dormant state because each cell found in young mice can be accounted for mathematically in aging mice. If permanent dormancy were not achieved after the fourth division, LR-HSCs could not accumulate to the extent they do with age. Together, this indicates that HSCs maintain a record of their cell divisions.

This data further allowed us to conclude that the LR-HSC population, which contains all LT-HSC activity, cannot be contributing to homeostatic hematopoiesis. This suggests that the populations that function to maintain the continuous supply of blood at steady state, and the populations that function as stem cells during transplantation are distinct. These findings are in agreement 
with recent work on homeostatic hematopoiesis (Busch et al., 2015; Sun et al., 2014), as well as on crypt cells in the gut (Buczacki et al., 2013) demonstrating that different populations act as stem cells in different regenerative contexts tissue maintenance and tissue regeneration after stress. Additionally, as our data indicate that single cell divisions within the LR-HSC compartment are capable of impacting on cell cycle entry, and in the case of a fifth division a complete loss of LT-HSC activity, not only are HSCs counting their divisions, but also single cell divisions can impact HSC behavior and regenerative potential.

\section{A mechanism of cell division counting may underlie age related changes to the HSC compartment}

Finally, we used our system to infer information about the hierarchical relationship of $\mathrm{CD} 41^{+}$and $\mathrm{CD} 41^{-} \mathrm{HSCs}$, both in the context of regeneration and homeostasis. While we initially thought that in aging mice the expanded CD41 $1^{+}$ HSC compartment would exclusively contain cells with myeloid-restricted regenerative potential, we found that in agreement with published data (Gekas and Graf, 2013), there are indeed $\mathrm{CD} 41^{+}$cells that exhibit $\mathrm{LT}-\mathrm{HSC}$ regenerative potential when assayed at the clonal level. These CD $41^{+}$LT-HSCs were found exclusively within the sLR-HSC compartment. Analysis of BM regenerated from retrospectively identified LT-HSCs after transplantation revealed that on the clonal level, both $\mathrm{CD} 41^{-}$and $\mathrm{CD} 41^{+} \mathrm{HSC}$ s had similar propensities to regenerate each other, suggesting that in the context of transplantation CD41 may not be an indicator of HSC hierarchical primitiveness. However, CD41 expression within the non-LR-HSC population did enrich for cells with myeloid-restricted 
regenerative potential, suggesting that $\mathrm{CD} 41$, and perhaps other cell surface markers, are greater predictors of cellular function once cells have left the labelretaining population. Interestingly, our label retention system also allowed us to make inferences about the hierarchical relationship of CD41 expressing cells within the LR-HSC. As GFP fluorescence intensity can only be lost over time in a cell division dependent manner, this enabled us to use the H2BGFP labelretaining system as a means to track the origin of expanding cell populations. Doing so demonstrated that CD41- LR-HSCs must give rise to the expanded CD $41^{+}$sLR-HSC population in aging mice.

It is important to state that while much of our evidence on homeostatic hematopoiesis is supportive of recent studies (Busch et al., 2015; Sun et al., 2014), and is supported by our mathematical models, further experiments need to be performed to fully validate these conclusions, possibly using a dual labelretaining and lineage-tracing genetic system that identifies progeny of labelretaining populations over time (Buczacki et al., 2013). Nevertheless, our findings, viewed overall, permit us to suggest a model for the aging of the HSC compartment (Figure 5) in which the dormant label-retaining compartment that contains all LT-HSC activity decreases in frequency within the HSC compartment but actually expands over time. This both explains the observation that aging HSCs demonstrate impaired function upon transplantation due to the diminished frequency of LR-HSCs within the stem cell compartment, while also revealing how functional HSCs increase with aging via increased absolute number of LRHSCs within the whole BM. This compartment symmetrically self-renews over 
time precluding contribution to homeostatic hematopoiesis, but each HSC can accumulate only 4 cell divisions while maintaining LT-HSC capacity. The non-LRHSC compartment also expands over time, but to a greater extent, and contains cells with limited self-renewal and differentiation capacity upon transplantation, which more likely supports homeostatic hematopoiesis. With aging, the non-LRHSC compartment becomes dominated by CD41 expressing cells that contain myeloid-restricted repopulation potential, and partially accounts for the increased propensity of the compartment as a whole to produce greater myeloid cell output with age. Although likely, it remains uncertain whether the non-LR-HSC compartment is directly derived from the LR-HSC compartment as a function of continuously accumulated proliferative history.

It will be interesting to investigate the underlying molecular mechanisms responsible for this cellular memory. Regulation of the number of stem and progenitor cell divisions greatly impacts on regeneration potential, and an inability to limit proliferation may lead to cancer progression. Several studies that have tracked cell division numbers in various systems ranging from Bacillus subtillis sporulation, to Drosophila spermatogenesis, to oligodendrocyte precursor differentiation, describe the accumulation over several rounds of division of a genetic factor that eventually reaches a threshold that either further divisions or initiates the onset of differentiation cascade (Dugas et al., 2007; Insco et al., 2009; Levine et al., 2012). In the context of our work, as we find cells enter permanent dormancy after 4 divisions and the initiation of a differentiation cascade upon the fifth cell division, we expect an inhibitor of proliferation and/or 
differentiation acts in a positive feedback loop to inhibit cell divisions without the addition of stress.

\section{Experimental Procedures}

\section{Mice}

Tg(tetO-HIST1H2BJ/GFP)47Efu (TetO-H2BGFP), hCD34-tTA (CD34), C57BL/6 (B6), and the congenic B6.SJL-Ptprc ${ }^{a} P e p c^{b} /$ BoyJ (SJL) mice were acquired and maintained as previously described (Qiu et al., 2014). Double transgenic mice 34/H2BGFP mice were derived from crossbreeding the single transgenic TetOH2BGFP and CD34 mice. F1 mice from this cross were used for all experiments, with the exception of cell cycle analysis, which was performed on B6 BM. Dox was administered through the drinking water at $1 \mathrm{mg} / \mathrm{ml}$ to mice beginning between 2-4 months of age. Dox water was changed twice weekly. Animal experiments were approved by the Institutional Animal Care and Use Committee and conducted in accordance with the Animal Welfare Act.

\section{Sample Preparation and Flow Cytometry}

BM cells were harvested from tibias, femurs, and pelvic bones by crushing with a mortar and pestle in a PBS buffer supplemented with $5 \%$ new born calf serum (NCS, GIBCO). Cells were triturated to obtain a single cell suspension, and bone debris was removed by filtering through a $70 \mu \mathrm{m}$ cell strainer (BD). Red cells were lysed with an ammonium chloride lysis buffer. For staining, cells were first incubated with biotin-conjugated lineage markers anti-CD3e, anti-B220, anti-Gr-1, anti-CD11b, and anti-Ter119, and on occasion also with biotin-conjugated anti- 
CD48 and anti-Flk2, followed by anti-biotin superparamagnetic beads (Dynabeads $®$ Biotin Binder, Life technologies), and lineage marker expressing cells were depleted via magnetic separation. The fraction enriched for Lineage ${ }^{-}$ ${ }^{\text {llow }}$ cells was further incubated with antibodies against CD150, CD48, c-Kit, Sca1, CD41, Flk2, IL-7R $\alpha, C D 34, F c \gamma R I I I$, and fluorophore-conjugated streptavidin. Dead cells were excluded by staining with 4', 6-diamidino-2-phenylindole (DAPI, Sigma), or propidium iodide (PI, Sigma). Cells were analyzed on an LSRII (Becton Dickenson) flow cytometer and sorted on an Influx (Becton Dickenson). Antibodies are listed in Table S3.

\section{Transplantation Assays}

HSCs were sorted from 34/H2B mice (CD45.2) that were 19 months of age, and had been chased with dox for 15 or 17 months into various populations based on label retention. Sorted cells from each population were injected retro-orbitally into lethally irradiated SJL (CD45.1) mice (2 rounds of 550 rads, three hours apart) at a dosage of 200 sorted cells plus $1.3 \times 10^{5}$ cells of Lin/CD48/Flk2-depleted competitor BM (CD45.1) per mouse. Mice were bled at timed intervals post transplantations from the retro-orbital venus plexus, red blood cells were lysed, and the contribution of donor derived CD $45.2^{+}$cells were assessed for contribution to the B cell $\left(B 220^{+}\right)$, T cell $\left(\mathrm{CD} 4 / \mathrm{CD} 8^{+}\right)$, and Myeloid $\left(\mathrm{CD} 11 \mathrm{~b} / \mathrm{Gr} 1^{+}\right)$ lineages. Granulocytes were identified as $\mathrm{SSC}^{\mathrm{Hi}} \mathrm{CD} 11 \mathrm{~b} / \mathrm{Gr} 1^{+}$cells. Secondary transplants were performed at 24 weeks post primary transplant and $5 \times 10^{6}$ cells of pooled whole BM from each group were transplanted into secondary hosts. 
Limiting dilution transplantations were performed by transplanting 15 sorted HSCs from 19-month old mice chased with dox for 17 months along with $2 \times 10^{5}$ cells of congenic un-manipulated competitor bone (CD45.1) into lethally irradiated young SJL mice. Secondary transplants were performed 24 weeks post primary transplantation by transplanting $5 \times 10^{6}$ cells of whole $B M /$ host from a single primary recipient mouse into two new hosts (CD45.1).

Additional Experimental Procedures available in the Supplemental Information online.

\section{Author Contributions:}

J.M.B. conceived and performed experiments, acquired and analyzed data, and wrote the manuscript. B.M. and H.S. developed the models, analyzed data, and edited the manuscript. H.S.K and X.N. performed experiments. K.A. conceived and performed experiments, edited the manuscript, and managed the project.

\section{Acknowledgements:}

The authors wish to thank members of the Moore and Lemischka laboratories for advice and criticisms, I. Lemischka, R. Brosh, S. Ghaffari, C. Schaniel, and R. Krauss for critical reading of the manuscript, and B. Dykstra for thoughtful discussions of the work. We also thank J. Gregory for her artistic contributions, and the flow cytometry and animal facility shared resources at the ISMMS. K.M. was supported by NIH 2R01HL58739 and J.M.B is supported by T32HD075735. 


\section{References:}

Ang, Y.S., Gaspar-Maia, A., Lemischka, I.R., and Bernstein, E. (2011). Stem cells and reprogramming: breaking the epigenetic barrier? Trends in pharmacological sciences 32, 394-401.

Beerman, I., Bhattacharya, D., Zandi, S., Sigvardsson, M., Weissman, I.L., Bryder, D., and Rossi, D.J. (2010). Functionally distinct hematopoietic stem cells modulate hematopoietic lineage potential during aging by a mechanism of clonal expansion. Proceedings of the National Academy of Sciences of the United States of America 107, 5465-5470.

Beerman, I., Bock, C., Garrison, B.S., Smith, Z.D., Gu, H., Meissner, A., and Rossi, D.J. (2013). Proliferation-dependent alterations of the DNA methylation landscape underlie hematopoietic stem cell aging. Cell stem cell 12, 413-425.

Beerman, I., Seita, J., Inlay, M.A., Weissman, I.L., and Rossi, D.J. (2014). Quiescent hematopoietic stem cells accumulate DNA damage during aging that is repaired upon entry into cell cycle. Cell stem cell 15, 37-50.

Benz, C., Copley, M.R., Kent, D.G., Wohrer, S., Cortes, A., Aghaeepour, N., Ma, E., Mader, H., Rowe, K., Day, C., et al. (2012). Hematopoietic stem cell subtypes expand differentially during development and display distinct lymphopoietic programs. Cell stem cell 10, 273-283.

Bowie, M.B., McKnight, K.D., Kent, D.G., McCaffrey, L., Hoodless, P.A., and Eaves, C.J. (2006). Hematopoietic stem cells proliferate until after birth and show a reversible phase-specific engraftment defect. The Journal of clinical investigation 116, 28082816.

Buczacki, S.J., Zecchini, H.I., Nicholson, A.M., Russell, R., Vermeulen, L., Kemp, R., and Winton, D.J. (2013). Intestinal label-retaining cells are secretory precursors expressing Lgr5. Nature 495, 65-69.

Burrill, D.R., and Silver, P.A. (2010). Making cellular memories. Cell 140, 13-18. Burrill, D.R., and Silver, P.A. (2011). Synthetic circuit identifies subpopulations with sustained memory of DNA damage. Genes \& development 25, 434-439. Busch, K., Klapproth, K., Barile, M., Flossdorf, M., Holland-Letz, T., Schlenner, S.M., Reth, M., Hofer, T., and Rodewald, H.R. (2015). Fundamental properties of unperturbed haematopoiesis from stem cells in vivo. Nature 518,542-546. Cho, R.H., Sieburg, H.B., and Muller-Sieburg, C.E. (2008). A new mechanism for the aging of hematopoietic stem cells: aging changes the clonal composition of the stem cell compartment but not individual stem cells. Blood 111, 5553-5561.

Dugas, J.C., Ibrahim, A., and Barres, B.A. (2007). A crucial role for p57(Kip2) in the intracellular timer that controls oligodendrocyte differentiation. The Journal of neuroscience : the official journal of the Society for Neuroscience 27, 6185-6196. Dykstra, B., Olthof, S., Schreuder, J., Ritsema, M., and de Haan, G. (2011). Clonal analysis reveals multiple functional defects of aged murine hematopoietic stem cells. The Journal of experimental medicine 208, 2691-2703. Efroni, S., Duttagupta, R., Cheng, J., Dehghani, H., Hoeppner, D.J., Dash, C., BazettJones, D.P., Le Grice, S., McKay, R.D., Buetow, K.H., et al. (2008). Global transcription in pluripotent embryonic stem cells. Cell stem cell 2, 437-447. 
Essers, M.A., Offner, S., Blanco-Bose, W.E., Waibler, Z., Kalinke, U., Duchosal, M.A., and Trumpp, A. (2009). IFNalpha activates dormant haematopoietic stem cells in vivo. Nature 458, 904-908.

Flach, J., Bakker, S.T., Mohrin, M., Conroy, P.C., Pietras, E.M., Reynaud, D., Alvarez, S., Diolaiti, M.E., Ugarte, F., Forsberg, E.C., et al. (2014). Replication stress is a potent driver of functional decline in ageing haematopoietic stem cells. Nature 512, 198202.

Foudi, A., Hochedlinger, K., Van Buren, D., Schindler, J.W., Jaenisch, R., Carey, V., and Hock, H. (2009). Analysis of histone 2B-GFP retention reveals slowly cycling hematopoietic stem cells. Nature biotechnology 27, 84-90.

Geiger, H., de Haan, G., and Florian, M.C. (2013). The ageing haematopoietic stem cell compartment. Nature reviews Immunology 13, 376-389.

Gekas, C., and Graf, T. (2013). CD41 expression marks myeloid-biased adult hematopoietic stem cells and increases with age. Blood 121, 4463-4472. Haas, S., Hansson, J., Klimmeck, D., Loeffler, D., Velten, L., Uckelmann, H., Wurzer, S., Prendergast, A.M., Schnell, A., Hexel, K., et al. (2015). Inflammation-Induced Emergency Megakaryopoiesis Driven by Hematopoietic Stem Cell-like Megakaryocyte Progenitors. Cell stem cell 17, 422-434. Harley, C.B., Futcher, A.B., and Greider, C.W. (1990). Telomeres shorten during ageing of human fibroblasts. Nature 345, 458-460. Insco, M.L., Leon, A., Tam, C.H., McKearin, D.M., and Fuller, M.T. (2009). Accumulation of a differentiation regulator specifies transit amplifying division number in an adult stem cell lineage. Proceedings of the National Academy of Sciences of the United States of America 106, 22311-22316.

Levine, J.H., Fontes, M.E., Dworkin, J., and Elowitz, M.B. (2012). Pulsed feedback defers cellular differentiation. PLoS biology 10, e1001252. Majumdar, A., Cesario, W.C., White-Grindley, E., Jiang, H., Ren, F., Khan, M.R., Li, L., Choi, E.M., Kannan, K., Guo, F., et al. (2012). Critical role of amyloid-like oligomers of Drosophila Orb2 in the persistence of memory. Cell 148, 515-529.

Mohrin, M., Bourke, E., Alexander, D., Warr, M.R., Barry-Holson, K., Le Beau, M.M., Morrison, C.G., and Passegue, E. (2010). Hematopoietic stem cell quiescence promotes error-prone DNA repair and mutagenesis. Cell stem cell 7, 174-185. Morrison, S.J., Wandycz, A.M., Akashi, K., Globerson, A., and Weissman, I.L. (1996). The aging of hematopoietic stem cells. Nature medicine 2,1011-1016. Muller-Sieburg, C.E., Cho, R.H., Karlsson, L., Huang, J.F., and Sieburg, H.B. (2004). Myeloid-biased hematopoietic stem cells have extensive self-renewal capacity but generate diminished lymphoid progeny with impaired IL-7 responsiveness. Blood 103, 4111-4118.

Muller-Sieburg, C.E., Cho, R.H., Thoman, M., Adkins, B., and Sieburg, H.B. (2002). Deterministic regulation of hematopoietic stem cell self-renewal and differentiation. Blood 100, 1302-1309.

Nakamura-Ishizu, A., Takizawa, H., and Suda, T. (2014). The analysis, roles and regulation of quiescence in hematopoietic stem cells. Development (Cambridge, England) 141, 4656-4666.

Pang, W.W., Price, E.A., Sahoo, D., Beerman, I., Maloney, W.J., Rossi, D.J., Schrier, S.L., and Weissman, I.L. (2011). Human bone marrow hematopoietic stem cells are 
increased in frequency and myeloid-biased with age. Proceedings of the National Academy of Sciences of the United States of America 108, 20012-20017.

Paul, F., Arkin, Y., Giladi, A., Jaitin, D.A., Kenigsberg, E., Keren-Shaul, H., Winter, D., Lara-Astiaso, D., Gury, M., Weiner, A., et al. (2015). Transcriptional Heterogeneity and Lineage Commitment in Myeloid Progenitors. Cell 163, 1663-1677.

Perie, L., Duffy, K.R., Kok, L., de Boer, R.J., and Schumacher, T.N. (2015). The Branching Point in Erythro-Myeloid Differentiation. Cell 163, 1655-1662. Pietras, E.M., Warr, M.R., and Passegue, E. (2011). Cell cycle regulation in hematopoietic stem cells. The Journal of cell biology 195, 709-720.

Qiu, J., Papatsenko, D., Niu, X., Schaniel, C., and Moore, K. (2014). Divisional history and hematopoietic stem cell function during homeostasis. Stem cell reports 2, 473490.

Rossi, D.J., Bryder, D., Seita, J., Nussenzweig, A., Hoeijmakers, J., and Weissman, I.L. (2007). Deficiencies in DNA damage repair limit the function of haematopoietic stem cells with age. Nature 447, 725-729.

Rossi, D.J., Bryder, D., Zahn, J.M., Ahlenius, H., Sonu, R., Wagers, A.J., and Weissman, I.L. (2005). Cell intrinsic alterations underlie hematopoietic stem cell aging. Proceedings of the National Academy of Sciences of the United States of America 102, 9194-9199.

Si, K., Giustetto, M., Etkin, A., Hsu, R., Janisiewicz, A.M., Miniaci, M.C., Kim, J.H., Zhu, H., and Kandel, E.R. (2003a). A neuronal isoform of CPEB regulates local protein synthesis and stabilizes synapse-specific long-term facilitation in aplysia. Cell 115, 893-904.

Si, K., Lindquist, S., and Kandel, E.R. (2003b). A neuronal isoform of the aplysia CPEB has prion-like properties. Cell 115, 879-891.

Sieburg, H.B., and Muller-Sieburg, C.E. (2004). Classification of short kinetics by shape. In silico biology 4, 209-217.

Sudo, K., Ema, H., Morita, Y., and Nakauchi, H. (2000). Age-associated characteristics of murine hematopoietic stem cells. The Journal of experimental medicine 192, 1273-1280.

Sun, J., Ramos, A., Chapman, B., Johnnidis, J.B., Le, L., Ho, Y.J., Klein, A., Hofmann, O., and Camargo, F.D. (2014). Clonal dynamics of native haematopoiesis. Nature 514, 322-327.

van der Wath, R.C., Wilson, A., Laurenti, E., Trumpp, A., and Lio, P. (2009). Estimating dormant and active hematopoietic stem cell kinetics through extensive modeling of bromodeoxyuridine label-retaining cell dynamics. PloS one 4, e6972.

Wilson, A., Laurenti, E., Oser, G., van der Wath, R.C., Blanco-Bose, W., Jaworski, M., Offner, S., Dunant, C.F., Eshkind, L., Bockamp, E., et al. (2008). Hematopoietic stem cells reversibly switch from dormancy to self-renewal during homeostasis and repair. Cell 135, 1118-1129.

Yamamoto, R., Morita, Y., Ooehara, J., Hamanaka, S., Onodera, M., Rudolph, K.L., Ema, H., and Nakauchi, H. (2013). Clonal analysis unveils self-renewing lineage-restricted progenitors generated directly from hematopoietic stem cells. Cell 154, 1112-1126. 


\section{Figure Legends:}

Figure 1: LR-HSCs Persist in BM Throughout Life and Contain All LT-HSC Activity in Aging BM. (A) Schematic of long-term dox treatments. 2-4 month old 34/H2BGFP mice were placed on dox for periods ranging from 3-22 months. At the end of dox chase BM was analyzed for the presence of LR-HSCs. (B) Histogram of LSK/CD48 $/ \mathrm{FIk2}^{-} / \mathrm{CD} 150^{+}$HSC compartment before and after 12 month dox chase. LR-HSCs were determined by gating above the background GFP levels of single transgenic TetO-H2BGFP HSCs. (C) Time course of label dilution after initiation of dox chase; $n=2-15$ mice per time point. (D) Percent of HSCs that are label-retaining after $10 \leq$ months of dox chase (sLR-HSCs). $n=42$ mice from 12 independent experiments. (E-L) HSC populations were sorted from 19 month-old mice chased with dox for 15 months into Total, GFP ${ }^{H i}$, and GFP ${ }^{\text {Lo }}$ HSC populations. 200 cells from each population were competitively transplanted to assay regenerative potential. (E) Gating strategy for Total, GFP ${ }^{\mathrm{Hi}}$, and GFP ${ }^{\mathrm{Lo}}$ HSC fractions. (F-G) Blood chimerism of granulocytes $(F)$, and total white blood cells $(\mathrm{G})$ during primary and secondary transplants. (H-J) Analysis of donorderived stem and progenitor cell compartments in recipient BM. Gating strategy $(\mathrm{H})$ and quantification of donor-derived HSPCs in primary $(\mathrm{I})$ and secondary $(\mathrm{J})$ transplantations after 22 and 24 weeks respectively. (K-L) Lineage distribution of donor-derived peripheral blood in primary $(\mathrm{K})$ and secondary $(\mathrm{L})$ hosts at 22 and 24 weeks respectively. Transplant data are represented as mean \pm SEM of 8-14 mice per group from two independent experiments. ${ }^{*} \mathrm{P}<0.05,{ }^{* *} \mathrm{P}<0.01$, ${ }^{* * *} \mathrm{P}<0.001$ by Welch's $t$ test. See also Figure $\mathrm{S} 1$. 
Figure 2: CD41 Expression on Young, Aging, and LR-HSCs. (A-D) FACS analysis and quantification of the primitive HSPC compartment from young and aging mice. (A) $\mathrm{CD} 150^{+}$cells marking the HSC compartment in young and aging BM. (B) Quantification of (A). (C) The same populations in (A) displayed as a function of CD150 and CD41 expression. (D) Quantification of (C). (E-G) CD41 expression on CD150 ${ }^{+} \mathrm{HSCs}$. (E) CD41 expression on Total, GFP ${ }^{\mathrm{Lo}}$, and GFP $\mathrm{Pi}^{\mathrm{Hi}}$ HSCs. (F) Quantification of CD41 expression on Total HSCs. (G) Ratio of CD41to $\mathrm{CD} 41^{+} \mathrm{HSCs}$ found in Total, GFP ${ }^{\mathrm{Lo}}$, and GFP ${ }^{\mathrm{Hi}} \mathrm{HSCs}$. Data are represented as mean \pm SEM of 9-11 mice per group from 3 independent experiments. ${ }^{* *} \mathrm{P}<0.01,{ }^{* * *} \mathrm{P}<0.001$ by Welch's $t$ test. See also Figure S2 and Figure S3.

Figure 3: Clonal Analysis of the Aging HSC Compartment Based on CD41 Expression and Label-Retention. HSCs were sorted from 19-month old mice chased with dox for 17 months into four populations based on CD41 expression and label retention, and transplanted at a dose of 15 cells per mouse. (A) Reconstitution curves for total white blood cells, myeloid, B cells, and T cells for each transplanted mouse through 24 weeks in primary and secondary recipients. The transition from primary to secondary transplantation is marked by the $\mathrm{x}$-axis break. The horizontal line marks the threshold of successful reconstitution. (B) Examples of the five reconstitution patterns observed. Definition of repopulation patterns: myeloid-restricted progenitors only repopulated myeloid cells; bipotent progenitors gave rise to myeloid and B cells; ST-HSCs showed transient repopulation of all three lineages, with donor chimerism of at least one lineage dropping below threshold by 24 weeks after primary transplantation; IT-HSCs 
repopulated all three lineages, but had at least one lineage drop below threshold by 24 weeks after secondary transplantation; and LT-HSCs maintained repopulation in all three lineages above threshold throughout both primary and secondary transplantation (C) Distribution of repopulating cell types found within each aging HSC compartment. The zoomed region represents $10 \%$ of the Total HSC compartment. (D) Heat maps displaying the regeneration of primitive BM populations by clonally transplanted aging HSCs after primary transplant. Transplanted cell populations are listed above each column—initially sorted cell type (left panel) and retrospectively categorized repopulating cell type (right panel). Regenerated HSPC types are listed to the right. The darker the chamber, the greater the proportion of reconstituted mice regenerated the given cell type. Numbers within each chamber represent the percentage in decimal format of reconstituted mice with each cell type. See also Figure S4.

\section{Figure 4: Dormant HSCs Count Symmetric Self-Renewal Divisions} Throughout Adult Life and Progress Towards Permanent Dormancy. (A-C) Analysis of H2BGFP subpopulations within the GFP ${ }^{H i}$ LR-HSC compartment. (A) Histogram displaying the H2BGFP peaks 0-4 visible within the LR-HSC compartment of young (3-4 months on dox) and aging mice (14-22 months on dox). Peak 0 corresponds to HSCs that have divided the least, while peak 4 corresponds to HSCs that have divided 4 more times than cells in peak 0. (B) Quantification of $(A) \cdot n=21$ and 13 mice from 6 independent experiments for young and aging mice, respectively. (C) Least squares fitting of single cell GFP intensity data collected from LR-HSCs found within each GFP peak of young 
mice. Observed experimental data is plotted as open circles, while predictions of a theoretical model in which H2BGFP concentration is reduced by a factor of 2 with each cell division is given by the dashed blue line. Experimental data was collected from 1568 single LR-HSCs from 6 independent experiments. (D-E) Percentage LR-HSCs within the HSC compartment (D) and absolute number of LR-HSCs per long bone in young and aging mice (E). Predicted LR-HSC numbers were generated by extrapolating the expansion of each young LR-HSC data point based on the distribution of cells found in peaks 0-4 for each mouse using the model in panel $(F)$, then corrected based on the average distribution of cells found in aging mice in panel (B). Data are mean \pm SEM of 17 and 10 mice from 5 and 3 independent experiments for young and aging mice respectively. (F) Symmetric self-renewal expansion model of LR-HSCs. As LR-HSCs slowly divide throughout adult life they transition from peak 0 to peak 4 , symmetrically self-renewing to double their numbers with each cell division. Arrows in the histogram depict the expansion capacity of cells as they progressively divide to reach peak 4. Numbers displayed are the average numbers of LR-HSCs in each peak per long bone. Boxed in red is the summation of LR-HSCs predicted to accumulate in peak 4 with aging. Numbers are the average of 21 young mice from 6 independent experiments. (G) Mathematical modeling of cell cycle progression as a function of divisional history within the LR-HSC compartment. Five models were considered (see Methods for details). Displayed are representations of cell cycle time progressions for each model (red dashed lines), as well as the experimentally determined (open circles) and model predicted 
sLR-HSC numbers (dashed blue curves) found in each GFP peak of aging mice. Cell cycle times for the step function and super-exponential models are actual times predicted by the model. As the constant, linear, and exponential models do not accurately predict the data, their corresponding cell cycle times are only visual representations. (H-I) Distribution of LR-HSCs across each GFP peak $(H)$, and quantification of LR-HSC absolute numbers after various lengths of dox chase (I). Legends refer to the length of dox chase. Data are representations of 2-6 independent experiments per group. (J) Cell cycle analysis of GFP Peak cells in young ( 5 months old, 3 month dox chase, $n=3$ ) and aging (11 months old, 9 month dox chase, $n=2$ ) mice. Each mouse represents an independent experiment. See also Figure S5.

\section{Figure 5: Self-Renewal Counting Model of Hematopoietic Stem Cell Aging.}

The HSC pool can be segregated into two populations based on label-retention. The LR-HSC pool contains all of the transplantable LT-HSC activity, while the non-LR-HSC pool is comprised of cells with minimal self-renewal and restricted regenerative capacity. With aging, both the LR- and non-LR-HSC pools expand. The LR-HSC pool undergoes four asynchronous symmetric self-renewal events, increasing the functional stem cell pool size over time while simultaneously diluting the GFP label with each cell division. After the fourth self-renewal event, LR-HSCs enter a state of permanent dormancy—as a fifth cell division would result in complete loss of LT-HSC potential-indicating that the LR-HSC population counts their cell divisions throughout life. The fact that the LR-HSC pool exclusively undergoes symmetric cell divisions means that they contribute 
minimally, if at all, to homeostatic hematopoiesis, unless activated to divide a fifth time by stress. The non-LR-HSC pool also expands with age, and represents the vast majority of the stem cell pool in aging mice. Within the non-LR-HSC pool $\mathrm{CD} 41^{+}$cells enriched for myeloid progenitor activity accumulate with time and dominate the aging HSC compartment. It is most likely that the non-LR-HSC pool maintains active hematopoiesis during steady state conditions. In the context of regeneration, we identified 5 types of stem and progenitor cells with regenerative potential after transplantation within the total HSC compartment. When analyzed as a total HSC population, the predominance of myeloid progenitors and cells with limited self-renewal potential contributes to increased myeloid representation in regenerated peripheral blood and reduced long-term engraftment. 


\section{Supplemental Figure Legends}

Figure S1 Related to Figure 1: Dynamic range and leakiness of the hCD34tTA x TetO-H2BGFP System. (A) Dynamic range of the H2BGFP reporter system in the absence of dox chase. Vertical lines indicate one-half dilutions in fluorescence intensity of the H2BGFP label indicating a range of 7-8 H2BGFP dilutions prior to reaching background level. (B) Experimental setup. Single transgenic hCD34-tTA and TetO-H2BGFP mice were mated while exposed to dox through the drinking water. Pups born from these matings were maintained on dox until adulthood, at which point BM was analyzed for the presence of H2BGFP expression above background levels. (C) Histogram showing GFP levels of LSKCD48-CD150+ cells from BM of 34/H2BGFP mice born on dox. (D) Modified experimental timeline. Mice born on dox were analyzed after a year of continuous dox treatment. (E) Histograms of GFP levels from three 34/H2BGFP mice born and maintained on dox for 1 year, and three single transgenic $\mathrm{H} 2 \mathrm{~B}$ mice (background). (F) Quantification of the brightest GFP intensity from each mouse displayed in (E).

Figure S2 Related to Figure 2: Quantification of Young and Aging HSC Populations, and Cell Cycle Analysis of HSCs based on CD41 Expression. (A-B) Frequency (A) and absolute number (B) of HSCs in young and aging bone marrow. $n=10-17$ mice per group. (C-D) Frequencies $(C)$ and absolute numbers (D) of various HSPC populations (I-III) in young and aging bone marrow. $n=6-7$ mice per group. (E-F) Frequency $(\mathrm{E})$ and absolute number $(\mathrm{F})$ of $\mathrm{CD} 41^{+} \mathrm{HSC}$ in young and aging bone marrow. $n=6-10$ mice per group. $(G-H)$ Frequencies $(G)$ and absolute number $(\mathrm{H})$ of HSC populations characterized based on CD41 expression and label retention in young and aging bone marrow. $n=6-10$ mice per group from 2-3 independent experiments. (I-J) Representative images (I) and quantification $(\mathrm{J})$ of $\mathrm{CD} 41^{-}$and $\mathrm{CD} 41^{+} \mathrm{HSC}$ static cell cycle profiles. $\mathrm{n}=6$ mice per group from two independent experiments. $(K)$ Histograms displaying the H2BGFP label retention over time of $\mathrm{CD} 41^{-}$and $\mathrm{CD} 41^{+} \mathrm{HSCs}$. Histograms are representations of young mice chased with dox for 12 weeks. (L) Quantification 
of H2BGFP label retention in (K). $n=9-11$ mice per group from three independent experiments. Data are represented as mean \pm SEM. ${ }^{*} P<0.05,{ }^{* *} P<0.01$, ${ }^{* * *} \mathrm{P}<0.001$ by Student $t$ test (quantifications), or paired Student $t$ test (cell cycle).

Figure S3 Related to Figure 2: Megakaryocyte Potential of HSC Compartment with Aging Based on Divisional History. Single GFP ${ }^{H i}$, GFP ${ }^{L O}$, and Total HSCs were sorted from young (5 months old, dox treated 3 months) and aging (11 months old, dox treated 9 months) into wells of a 96 well plate and were cultured in the presence of SCF, IL-3, and Tpo. (A-D) Images of representative colonies after 13 days in culture. Mixed cells colonies that contained both small and large cells (A and B), small cell only colonies (C), and large cell only colonies (D). Yellow arrows mark large megakaryocyte-like cells. $(E-F)$ Representative images of cytospun mixed (E) and small cell only colonies (F) stained with H\&E. Only mixed colonies showed megakaryocytes with large multi-lobed nuclei (black arrows). Large cell only colonies generated too few cells to be mounted on slides for staining. (G) Quantification of colony types found from each sorted HSC population. $(\mathrm{H})$ Quantification of colony size at day 13 generated from each sorted HSC population. Data are represented as mean \pm SEM of 64-130 single cells per group from 4 independent experiments.

\section{Figure S4 Related to Figure 3: Synchronistic Repopulation Kinetics in} Paired Secondary Transplantations. Bone marrow from each mouse repopulated with 15 cells from aging HSC populations was transplanted into paired secondary hosts. Repopulation kinetics were followed in both secondary recipients over 24 months to determine the degree of synchronicity of total white blood cell repopulation (\%CD45.2 $\left.{ }^{+}\right)$in independent hosts. We quantitatively defined the degree of synchronicity as the Hamming distance between pairs of time series. (A) Repopulation curves were grouped into 2 clusters based on the degree of synchronicity. The cluster boxed in grey contains curves with kinetics determined to be synchronous, while the cluster boxed in red contains asynchronous repopulation for paired secondary hosts. The letter on the right 
side of each repopulation kinetic indicates the retrospectively identified repopulating cell type. L, LT-HSC; I, IT-HSC; S, ST-HSC, B, Bipotent Progenitor, M, Myeloid Progenitor. (B) Scaled paired secondary repopulation curves used to determine symbolic dynamics data from each secondary repopulation curve. The orange and blue curves represent individual secondary recipients. Shaded plots indicate asynchronous repopulation behavior. (C) Hamming distance measurements. Any two kinetics were defined to be asynchronous (red dots), if their Hamming distance was $>2$. One exception occurred where secondary repopulation was considered asynchronous with a Hamming distance of 2 due to a shorter symbolic dynamic sequence.

Figure S5 Related to Figure 4: Cell Cycle Profiles of LR-HSCs and Young CD41- LR-HSCs Generate CD41 ${ }^{+}$sLR-HSCs in Aging Mice. (A) Cell cycle analysis of GFP ${ }^{H i}$ HSCs across each GFP peak in young (5 months old, 3 months dox) and aging mice (11 months old, 9 months dox). Between $5000-$ 15000 events in the Total HSC gate were acquired for each sample. $n=3$ and 2 for Young and Aging mice respectively. (B) Distributions of $\mathrm{CD} 41^{-}$(left) and $\mathrm{CD} 41^{+}$(right) LR-HSCs in young and aging mice across GFP peaks 0-4. (C) Representative histograms directly comparing GFP levels in CD41 $1^{-}$and CD41 ${ }^{+}$ LR-HSCs from young (left) and aging (right) mice. (D) GFP mean fluorescence intensity (MFI) of CD41 $1^{-}$and CD41 $1^{+}$LR-HSCs in young and aging mice. $n=9-11$ mice per group from 3 independent experiments. (E) Quantification of CD41' and CD $41^{+}$LR-HSCs in young and aging mice. Predicted expansion capacity of young HSCs as predicted by the model in Figure 4F. $n=6-7$ mice from 2 independent experiments. (F) Mathematical modeling of CD41- LR-HSC contribution to the CD $41^{+}$LR-HSC compartment. Predictions of a model in which the cell cycle time extends super-exponentially with the number of cell divisions (see Figure 4G) and in which CD41- LR-HSCs gain CD41 expression with probability $\alpha$ each time they divide are given in blue (full model details in Methods). This model most accurately fits the data when $\alpha=0.12$, suggesting that approximately 1 in every 10 CD $41^{-}$LR-HSC divisions gives rise to a CD41 ${ }^{+}$ 
daughter cell. Data are displayed as mean \pm SEM ${ }^{*} P<0.05,{ }^{* *} P<0.01,{ }^{* * *} \mathrm{P}<0.001$

by Welch's $t$ test or paired $t$ test.

Table S1 Related to Figure 1: Peripheral Blood Reconstitution by Aging HSCs.

Table S2 Related to Figure 4: Limiting Dilution Analysis.

Table S3 Related to Experimental Procedures: Antibody Panel. 


\section{Supplemental Experimental Procedures}

\section{HSPC Definitions}

The various stem and progenitor populations were defined as follows: HSCs ( $\mathrm{Lin}^{-}$ /Sca- $1^{+} / \mathrm{C}-\mathrm{Kit}^{+} / \mathrm{CD} 48^{-} / \mathrm{Flk} 2^{-} / \mathrm{CD} 150^{+}$or $\mathrm{Lin}^{-} / \mathrm{Sca}-1^{+} / \mathrm{c}-\mathrm{Kit}^{+} / \mathrm{CD} 48^{-} / \mathrm{CD} 150^{+}$), MPPs ( $\left.\mathrm{Lin}^{-} / \mathrm{Sca}-1^{+} / \mathrm{c}-\mathrm{Kit}^{+} / \mathrm{CD} 48^{-} / \mathrm{CD} 150^{-}\right), \mathrm{HPC}-1$ ( $\left.\mathrm{Lin}^{-} / \mathrm{Sca}-1^{+} / \mathrm{c}-\mathrm{Kit}^{+} / \mathrm{CD} 48^{+} / \mathrm{CD} 150^{-}\right)$, HPC-2 ( $\left.\mathrm{Lin}^{-} / \mathrm{Sca}-1^{+} / \mathrm{C}-\mathrm{Kit}^{+} / \mathrm{CD} 48^{+} / \mathrm{CD} 150^{+}\right)$, MkPs (Lin ${ }^{-} / \mathrm{Sca}-{ }^{-} / \mathrm{C}-$ $\left.\mathrm{Kit}^{+} / \mathrm{CD} 150^{+} / \mathrm{CD} 41^{+}\right)$, CMPs (Lin $\left./ \mathrm{Sca}-1^{-} / \mathrm{c}-\mathrm{Kit}^{+} / \mathrm{CD} 34^{+} / \mathrm{Fc} \gamma \mathrm{RIII}{ }^{-}\right), \mathrm{GMPs}\left(\mathrm{Lin}^{-} / \mathrm{Sca}-\right.$ $\left.1^{-} / \mathrm{c}-\mathrm{Kit}^{+} / \mathrm{CD} 34^{+} / \mathrm{Fc} \gamma \mathrm{RIII}{ }^{+}\right)$, MEPs ( $\left.\mathrm{Lin}^{-} / \mathrm{Sca}-1^{-} / \mathrm{c}-\mathrm{Kit}^{+} / \mathrm{CD} 34^{-} / \mathrm{Fc} \gamma \mathrm{RIII}{ }^{-}\right), \mathrm{CLPs}\left(\mathrm{Lin}^{-}\right.$

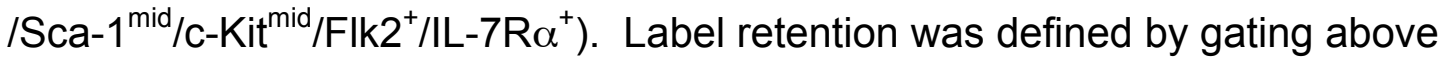
the background GFP levels found in heterozygous single transgenic TetOH2BGFP HSCs.

\section{Limiting Dilution Analysis}

Limiting dilution analysis was performed with peripheral blood reconstitution data at 24 weeks after primary transplant using the freely available ELDA software (http://bioinf.wehi.edu.au/software/elda/).

\section{Cell Cycle Analysis}

Cells were stained and prepared as above, then fixed in $4 \%$ methanol-free paraformaldehyde diluted in PBS. Cells were then washed three times with PBS containing $5 \%$ NCS, permeabilized in $0.2 \%$ Triton-X 100 , then stained with anti$\mathrm{Ki}-67$ (PE, eBioscience), and DAPI prior to analysis.

\section{Hamming Distance Analysis}

The Hamming distance was measured for pairs of leukocyte repopulation kinetics from secondary recipient mice as described previously (Müller-Sieburg et al., 2002). Thereby, the numerical kinetics of total peripheral blood white blood cell chimerism from paired secondary recipients were first transformed into symbolic dynamics based on the slopes of consecutive pairs of donor-type cell values over time as described. Symbolic dynamics are defined as sequences of symbols from the sign of the slope ("+" = positive slope, "-" = negative slope, " $\sim$ " = close to 
zero slope). Next, the Hamming distances of all pairs of symbolic dynamics were determined. Pairs of secondary transplant kinetics were defined as synchronous if their Hamming distance was below an empirically set threshold (the smallest possible Hamming distance $=0$ indicated that the two kinetics were identical based on this clustering measure). All pairs of kinetics with Hamming distances above the threshold were considered asynchronous.

\section{Mathematical Modeling}

Assuming that GFP dilutes by a factor of 2 with each cell division, the relative positions of the GFP peaks in the LR-HSC population are described by the following model:

$$
y_{n}=\left(\frac{y_{0}-c}{2^{n}}\right)+c,
$$

where $y_{n}$ is the mean fluorescence intensity at the $n^{\text {th }}$ peak and $c$ is a constant that accounts for background fluorescence. The MFI of GFP levels in HSCs of single transgenic TetO-H2BGFP mice was used to estimate $c$.

Expansion of LR-HSC numbers during aging was described by the following model:

$$
\begin{aligned}
& \frac{d x_{0}}{d t}=-k_{0} x_{0}, \\
& \frac{d x_{1}}{d t}=2 k_{0} x_{0}-k_{1} x_{1}, \\
& \frac{d x_{2}}{d t}=2 k_{1} x_{1}-k_{2} x_{2}, \\
& \frac{d x_{3}}{d t}=2 k_{2} x_{2}-k_{3} x_{3}, \\
& \frac{d x_{4}}{d t}=2 k_{3} x_{3}-k_{4} x_{4},
\end{aligned}
$$

where $x_{n}(t)$ is the expected number of cells per long bone that have divided $n$ (= $0,1,2,3,4)$ times since dox chase, and $\ln (2) / k_{n}$ is the expected length of time to the next division for a cell that has previously divided $n$ times. The above 
system is linear and homogeneous and may therefore be directly integrated and easily compared with experimental data. To determine how divisional history affects cell cycle time we fit the following functional forms for $k_{n}$ to experimental data using nonlinear least squares fitting.

1. Let $k_{n}=k$ for all $n$. In this model, cells cycle on average at the same rate regardless of how many times they have divided previously. In this case there is one free parameter $k=\ln (2) / \tau$, where $\tau$ is the expected cell cycle time.

2. Let $k_{n}=k$ for $n<4$ and $k_{4}=0$. In the first model $x_{n} \rightarrow 0$ as $t \rightarrow \infty$ for all $n$ (i.e. GFP ultimately dilutes away in all cells). However, in this modification $x_{n} \rightarrow 0$ as $t \rightarrow \infty$ for $n<4$ and $x_{4} \rightarrow 16 x_{0}(0)+8 x_{1}(0)+4 x_{2}(0)+2 x_{3}(0)+$ $x_{4}(0)$, where $x_{n}(0)$ is the initial number of cells in state $n$. This model assumes the cells proliferate normally until they have undergone four divisions, at which point they adopt a permanently quiescent state.

3. Let $k_{n}=k_{0}+n \beta$ for all $n$. In this model the cell cycle time grows linearly with divisional history: each cell cycle is, on average, $\beta$ hours longer than the last.

4. Let $k_{n}=k / \beta^{n}$, with $\beta>1$ for all $n$. In this model the expected cell cycle time grows exponentially with divisional history: each cell cycle is, on average, $\beta$ times longer than the last.

5. Let $k_{n}=k / \beta^{2^{n}}$ with $\beta>1$ for all $n$. In this model the expected cell cycle time grows super-exponentially as the cells divide: then the cell cycle is, on average, $\beta^{2^{n}}$ times longer than the $(n-1)^{\text {th }}$.

6. Let $k_{n}=k^{2^{n}}$ for $k<1$. In this model the expected cell cycle time also grows super- exponentially as the cells divide: the $n$th cell cycle is, on average, $k^{-2^{n}}$, $\mathrm{k}^{-2^{\mathrm{n}}}$ times longer than the $(n-1)^{\text {th }}$.

To account for the gain of CD41 expression in the LR-HSC fraction during aging, 
we assume that CD41 negative LR-HSCs gain CD41 expression with probability $\alpha$ each time they divide (i.e. we view gain of CD41 expression as marking a differentiation event that occurs through cell division). The dynamics of CD41 expression within the LR fraction are then described by the following model:

$$
\begin{aligned}
\frac{d x_{0}}{d t} & =-k_{0} x_{0}, & \frac{d y_{0}}{d t} & =-k_{0} y_{0}, \\
\frac{d x_{1}}{d t} & =2 \alpha k_{0} x_{0}-k_{1} x_{1}, & \frac{d y_{1}}{d t} & =2 k_{0} x_{0}+2(1-\alpha) k_{0} x_{0}-k_{1} x_{1}, \\
\frac{d x_{2}}{d t} & =2 \alpha k_{1} x_{1}-k_{2} x_{2}, & \frac{d y_{2}}{d t} & =2 k_{1} x_{1}+2(1-\alpha) k_{1} x_{1}-k_{2} x_{2}, \\
\frac{d x_{3}}{d t} & =2 \alpha k_{2} x_{2}-k_{3} x_{3}, & \frac{d y_{3}}{d t} & =2 k_{2} x_{2}+2(1-\alpha) k_{2} x_{2}-k_{3} x_{3}, \\
\frac{d x_{4}}{d t} & =2 \alpha k_{3} x_{3}-k_{4} x_{4}, & \frac{d y_{4}}{d t} & =2 k_{3} x_{3}+2(1-\alpha) k_{3} x_{3}-k_{4} x_{4},
\end{aligned}
$$

where $x_{n}(t)$ and $y_{n}(t)$ are the expected number of CD41 $1^{-}$and CD $41^{+}$LR-HSCs cells per long bone that have divided $n(=0,1,2,3,4)$ times since dox chase. To minimize the number of free parameters we assumed that CD41 status does not alter cell cycle progression and use model 5 , above, to account for the onset of cellular quiescence. In this case the full model has three free parameters $(k, \beta$, and $\alpha$ ). 


\section{Supplemental Tables}

\begin{tabular}{|c|c|c|c|c|c|}
\hline \multirow[b]{2}{*}{$\begin{array}{c}\text { Primary } \\
\text { Transplant }\end{array}$} & \multirow[b]{2}{*}{$\%$ CD45.2+ } & \multirow[b]{2}{*}{$\begin{array}{c}\text { \% CD45.2+ } \\
\text { Granulocytes }\end{array}$} & \multicolumn{3}{|c|}{$\%$ of Peripheral Blood } \\
\hline & & & B Cells & T Cells & Myeloid \\
\hline Total & $20.97 \pm 5.16$ & $35.24 \pm 7.96$ & $9.74 \pm 3.09$ & $3.34 \pm 1.90$ & $6.96 \pm 1.55$ \\
\hline GFPHi & $30.25 \pm 6.04$ & $64.60 \pm 6.27$ & $14.01 \pm 3.50$ & $2.66 \pm 1.47$ & $12.50 \pm 1.77$ \\
\hline GFPLo & $10.07 \pm 3.16$ & $22.56 \pm 6.57$ & $5.85 \pm 2.38$ & $0.91 \pm 0.38$ & $2.96 \pm 0.95$ \\
\hline \multicolumn{6}{|l|}{$\begin{array}{l}\text { Secondary } \\
\text { Transplant }\end{array}$} \\
\hline Total & $14.55 \pm 2.87$ & $20.54 \pm 7.98$ & $5.97 \pm 1.33$ & $4.06 \pm 1.29$ & $4.28 \pm 1.65$ \\
\hline GFPHi & $12.03 \pm 3.95$ & $29.70 \pm 7.58$ & $11.61 \pm 5.79$ & $1.13 \pm 0.46$ & $4.04 \pm 1.11$ \\
\hline GFPLo & $1.30 \pm 0.65$ & $1.40 \pm 0.49$ & $0.80 \pm 0.41$ & $0.26 \pm 0.21$ & $0.20 \pm 0.06$ \\
\hline \multicolumn{6}{|c|}{$\begin{array}{l}\text { Table S1 - Related to Figure } 1 \text {. Donor cell contribution to peripheral blood. } \\
\text { White blood cell and granulocyte contributions, as well as donor derived lineage } \\
\text { contributions to total peripheral blood at the end of primary ( } 22 \text { weeks) or } \\
\text { secondary ( } 24 \text { weeks) transplantation. Data are represented as mean } \pm \text { SEM of } \\
8-14 \text { mice per group from two independent experiments. }\end{array}$} \\
\hline
\end{tabular}




\begin{tabular}{|c|c|c|c|c|c|c|}
\hline & $\begin{array}{c}\text { Mice } \\
\text { Reconstituted }\end{array}$ & $\begin{array}{l}\text { Repopulating } \\
\text { Cell Frequency } \\
\quad(1 / \mathbf{x})\end{array}$ & $\begin{array}{l}\text { Repopulating } \\
\text { Cell Range } \\
(95 \% \mathrm{Cl})\end{array}$ & $\begin{array}{l}\text { Cell \#I } \\
\text { Mouse }\end{array}$ & $\begin{array}{l}\text { Cell \# } \\
\text { Range } \\
(95 \% \mathrm{Cl})\end{array}$ & $\begin{array}{l}\text { CD45.2 } \\
\text { Chimerism } \\
(\% \pm \text { SEM) }\end{array}$ \\
\hline $\begin{array}{l}\text { GFPLO } \\
\text { CD41 }^{+}\end{array}$ & $6 / 12$ & 21.6 & $9.57<x<49.0$ & 0.69 & $1.57>x>0.31$ & $1.12 \pm 0.67 \%$ \\
\hline $\begin{array}{l}\text { GFPLo } \\
\text { CD41 }\end{array}$ & $7 / 11$ & 14.8 & $6.85<x<32.1$ & 1.01 & $2.19>x>0.47$ & $13.09 \pm 11.73 \%$ \\
\hline $\begin{array}{l}\text { GFPHi }^{H i} \\
\text { CD41 }^{+}\end{array}$ & $4 / 6$ & 13.7 & $4.87<x<38.2$ & 1.09 & $3.08>x>0.39$ & $37.59 \pm 19.55 \%$ \\
\hline $\begin{array}{l}\text { GFPHi } \\
\text { CD41. }\end{array}$ & $7 / 9$ & 9.97 & $4.42<x<22.5$ & 1.50 & $3.39>x>0.67$ & $27.70 \pm 6.62 \%$ \\
\hline \multicolumn{7}{|c|}{$\begin{array}{l}\text { Table S2 - Related to Figure 3. Limiting dilution analysis of aging HSC populations. CD } 45.2^{+} \\
\text {chimerism was determined at } 24 \text { weeks in primary transplantation, and averaged between all mice } \\
\text { considered repopulated regardless of retrospectively identified repopulating cell type. }\end{array}$} \\
\hline
\end{tabular}




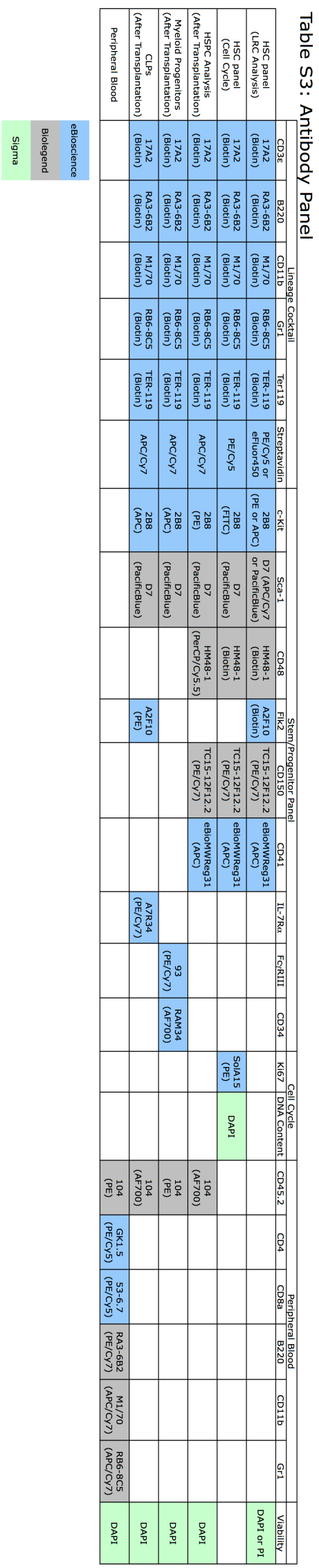




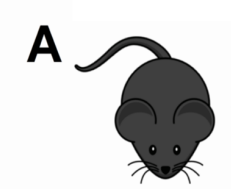

2-4 months $\mathrm{t}=0$
Young mice

(5-7 months old)
Aging mice

(18-25 months old)

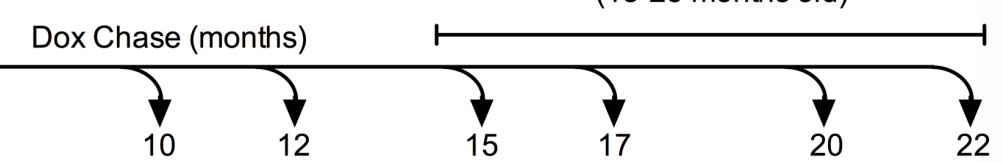

$\mathrm{BM}$ analysis at various time points after dox treatment

B

No Chase

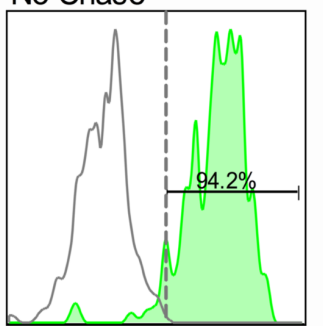

GFP

E LSKCD48-FIK2-

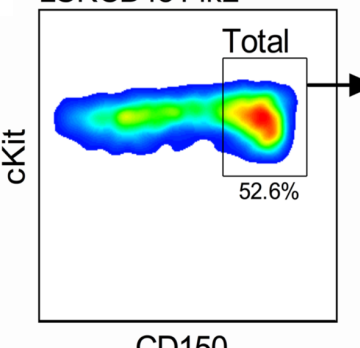

CD150

F

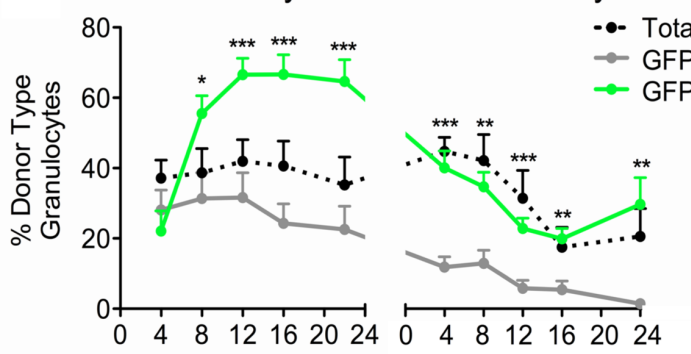

Weeks

G

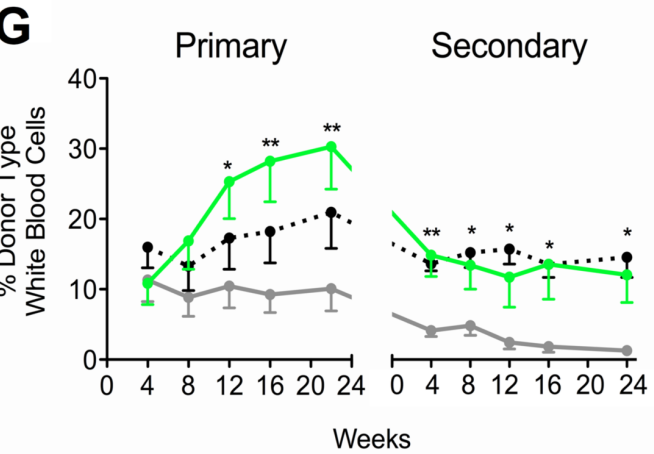

12 Month Chase
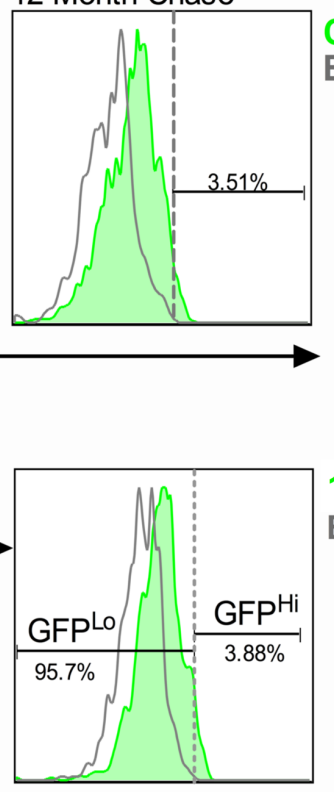

GFP
15 Months Dox Background

C

CD34 x H2BGFP Background

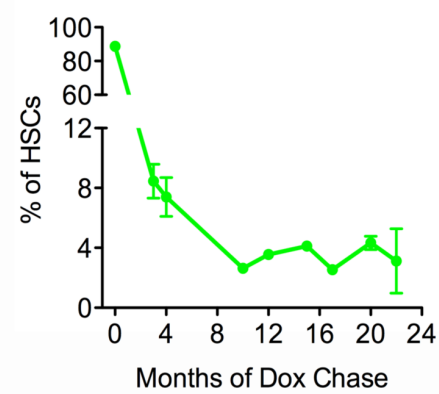

H

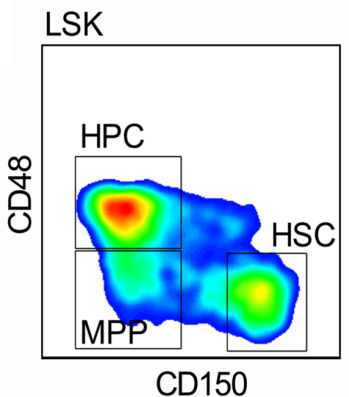

D

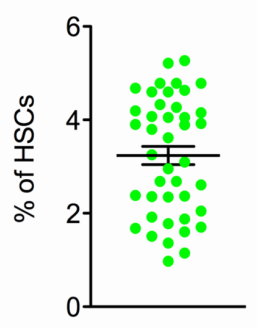

I

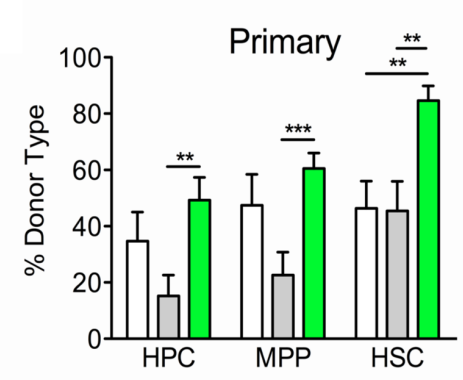

Primary

K

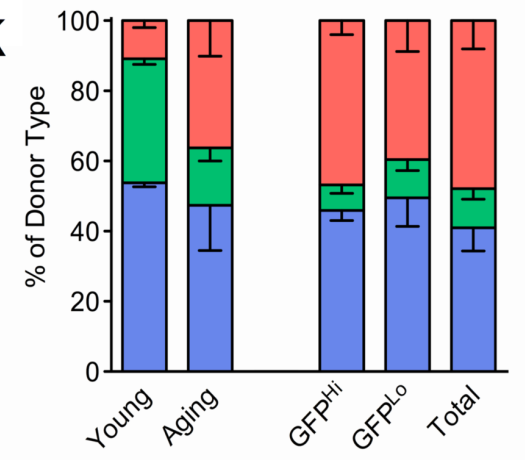

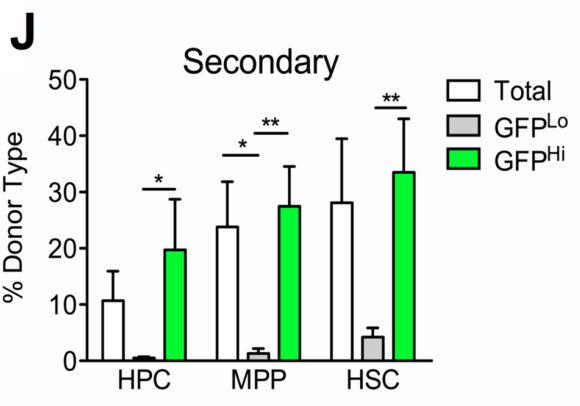

Secondary

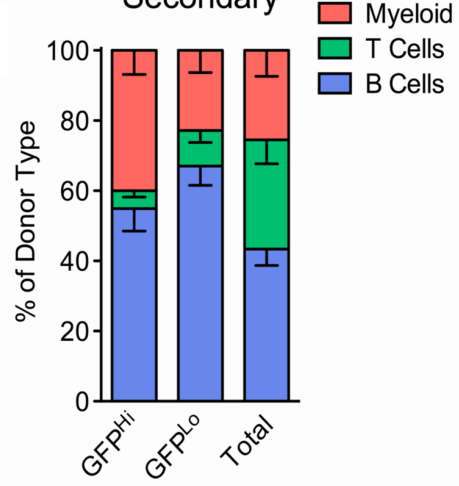

Figure 1 
A

Aoung
LSKCD48'FIK2:

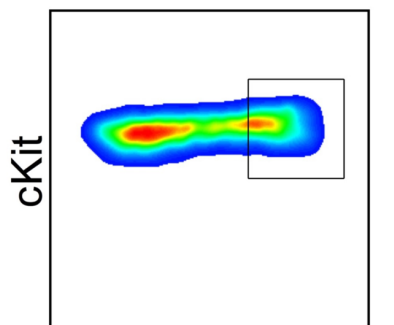

CD150

C Young

LSKCD48FIk2:

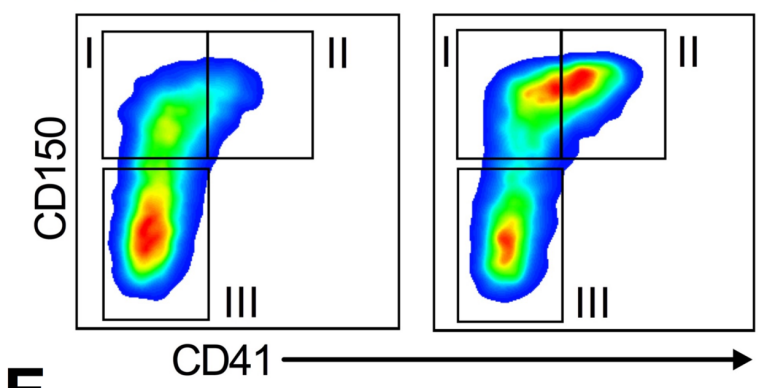

E

LSKCD48-FIk2-

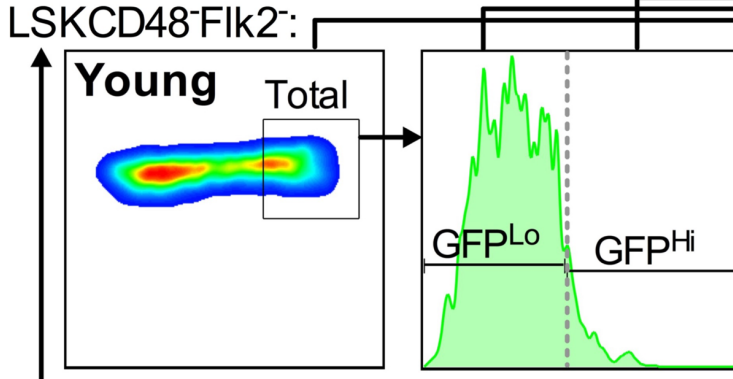

Aging $\quad$ Total

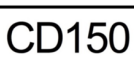

F

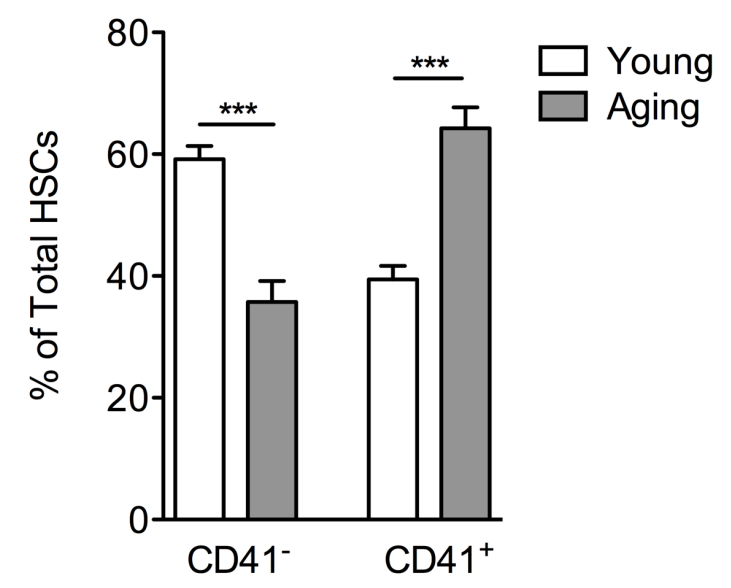

B



D

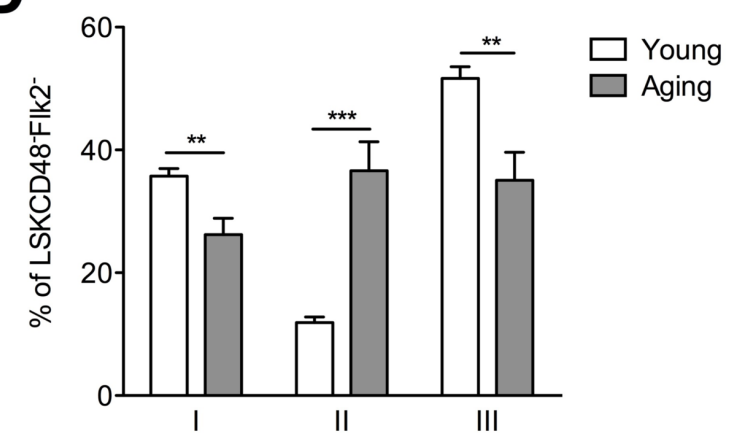

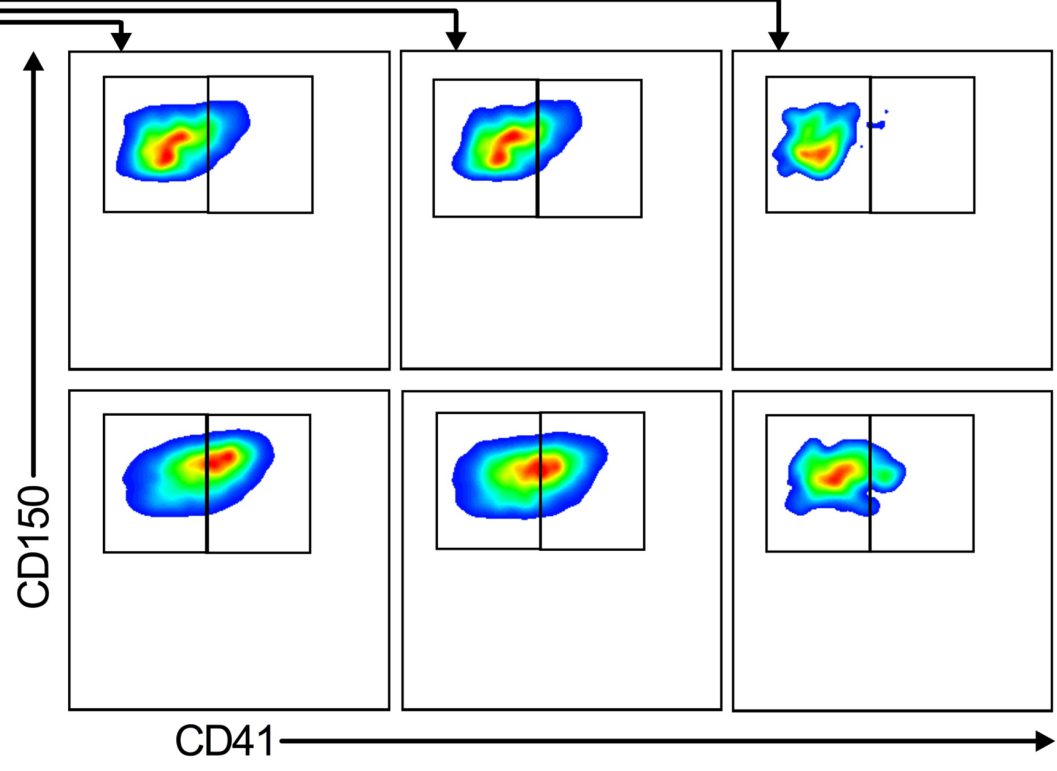

G

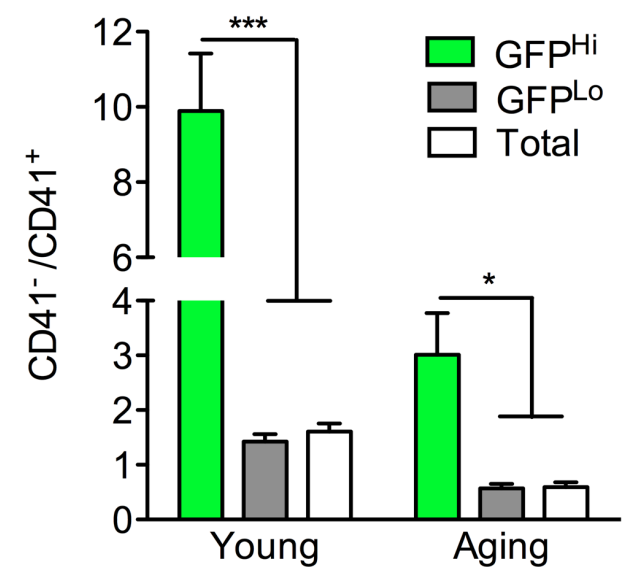



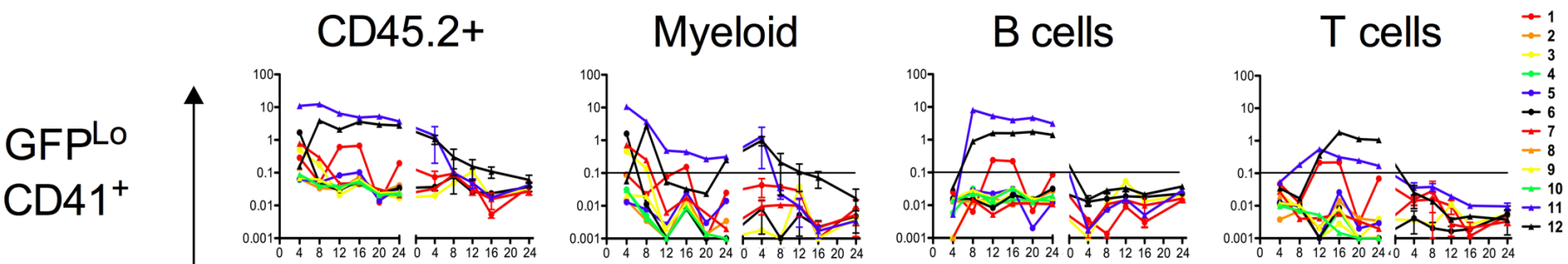

GFPLo

CD41-
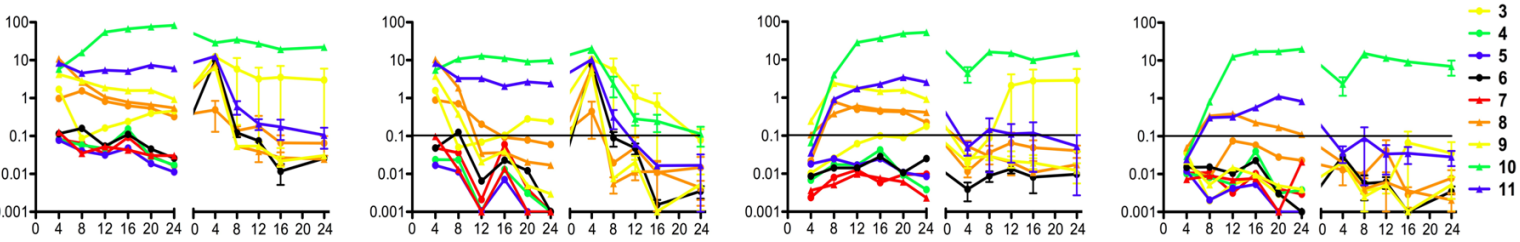

GFPHi

CD41 ${ }^{+}$
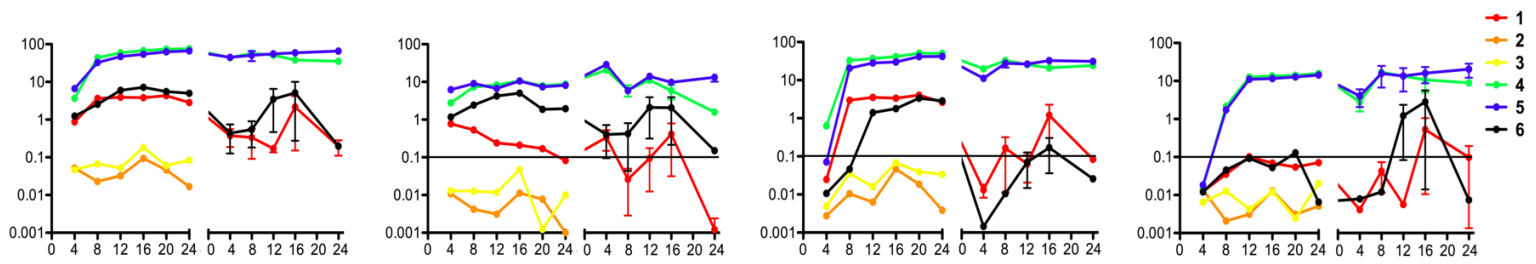

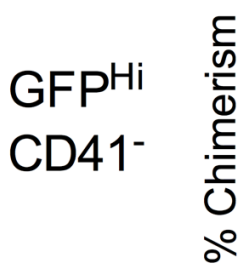
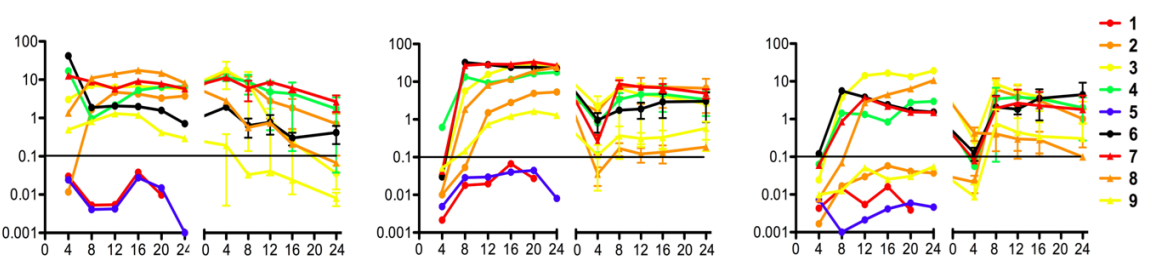

Weeks

B

Myeloid Restricted

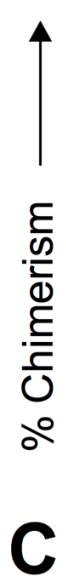

$\frac{0}{0}$

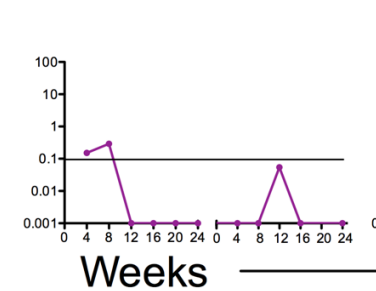

Bipotent Progenitor

ST-HSC

IT-HSC

LT-HSC
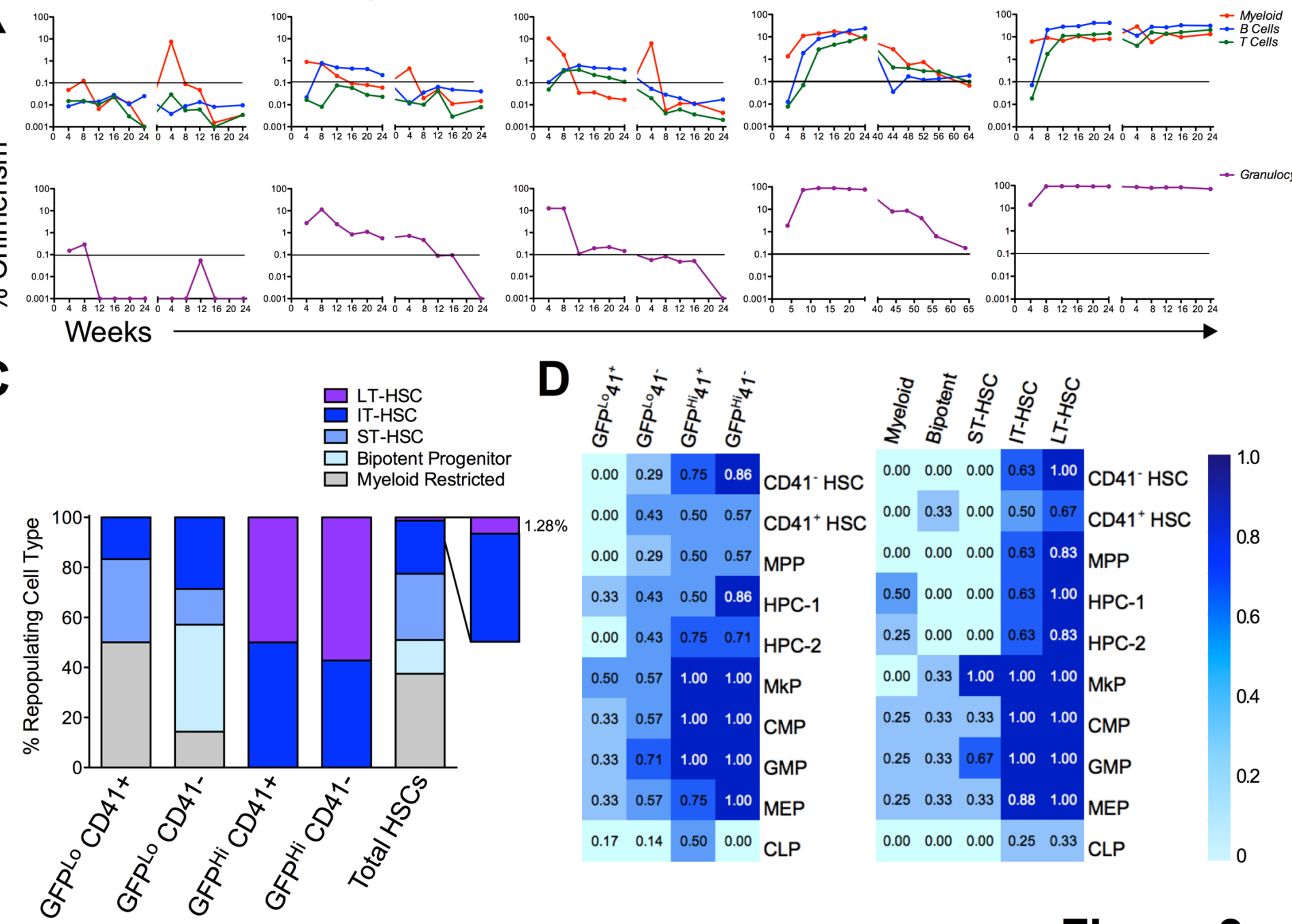



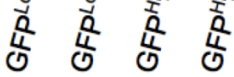
$0.00 \quad 0.29 \quad 0.75 \quad 0.86$ CD 41 - HSC $0.00 \quad 0.00 \quad 0.000 .63 \quad 1.00$ CD41- HSC

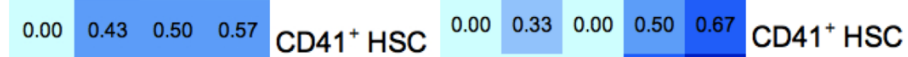
$\begin{array}{llllllllllllll}0.00 & 0.29 & 0.50 & 0.57 & \text { MPP } & & 0.00 & 0.00 & 0.00 & 0.63 & 0.83 & \text { MPP }\end{array}$ $\begin{array}{lllll}0.33 & 0.43 & 0.50 & 0.86 & \text { HPC- } 1\end{array}$ $\begin{array}{lllll}0.00 & 0.43 & 0.75 & 0.71 & \text { HPC-2 }\end{array}$ $\begin{array}{llll}0.50 & 0.57 & 1.00 & 1.00 \\ M k P\end{array}$ $\begin{array}{lllll}0.33 & 0.57 & 1.00 & 1.00 & \text { CMP }\end{array}$ $\begin{array}{llll}0.33 & 0.71 & 1.00 & 1.00\end{array}$ GMP $\begin{array}{lllll}0.33 & 0.57 & 0.75 & 1.00 & \text { MEP }\end{array}$ $\begin{array}{lllll}0.17 & 0.14 & 0.50 & 0.00 & \text { CLP }\end{array}$

Granulocytes 


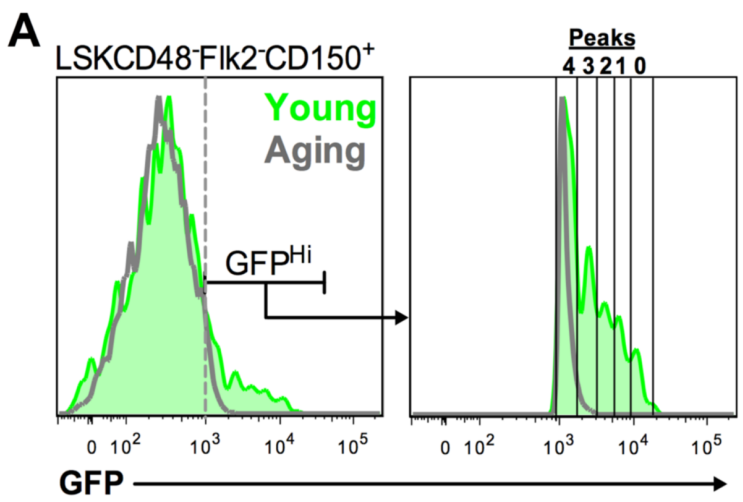

B

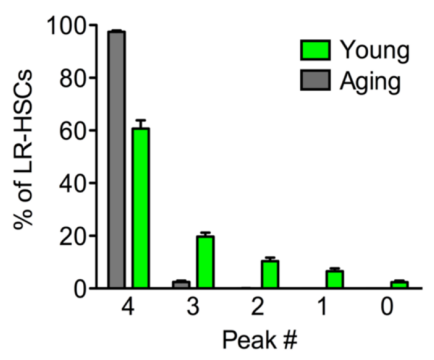

C

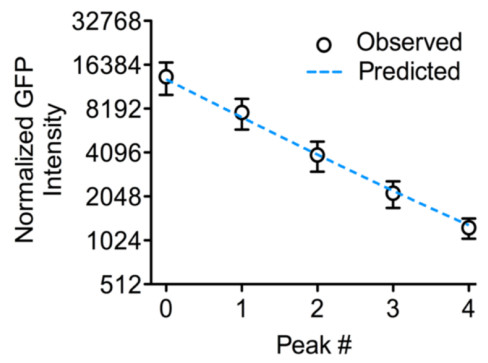

D

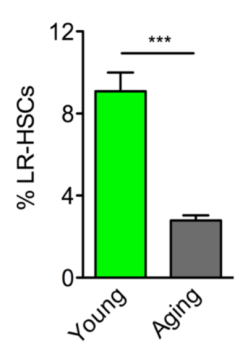

E

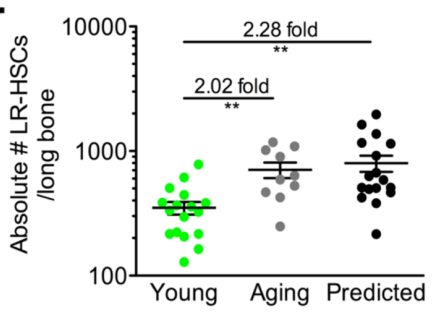

F $\frac{\text { Peaks }}{43210}$

$16 x$

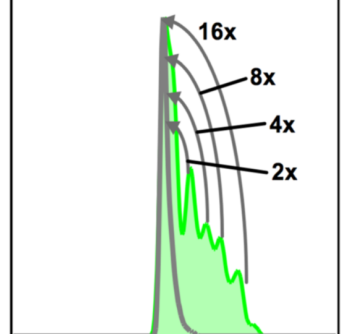

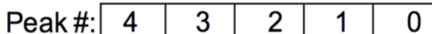

\begin{tabular}{|c|c|c|c|c|c|}
\hline \# of divisions to Peak 4: & 0 & 1 & & & \\
\hline
\end{tabular}

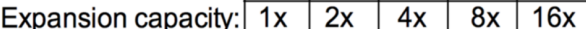

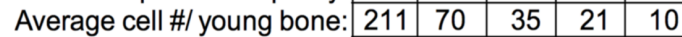

Predicted cell \#/ aging bone: $211+140+140+168+160=819$
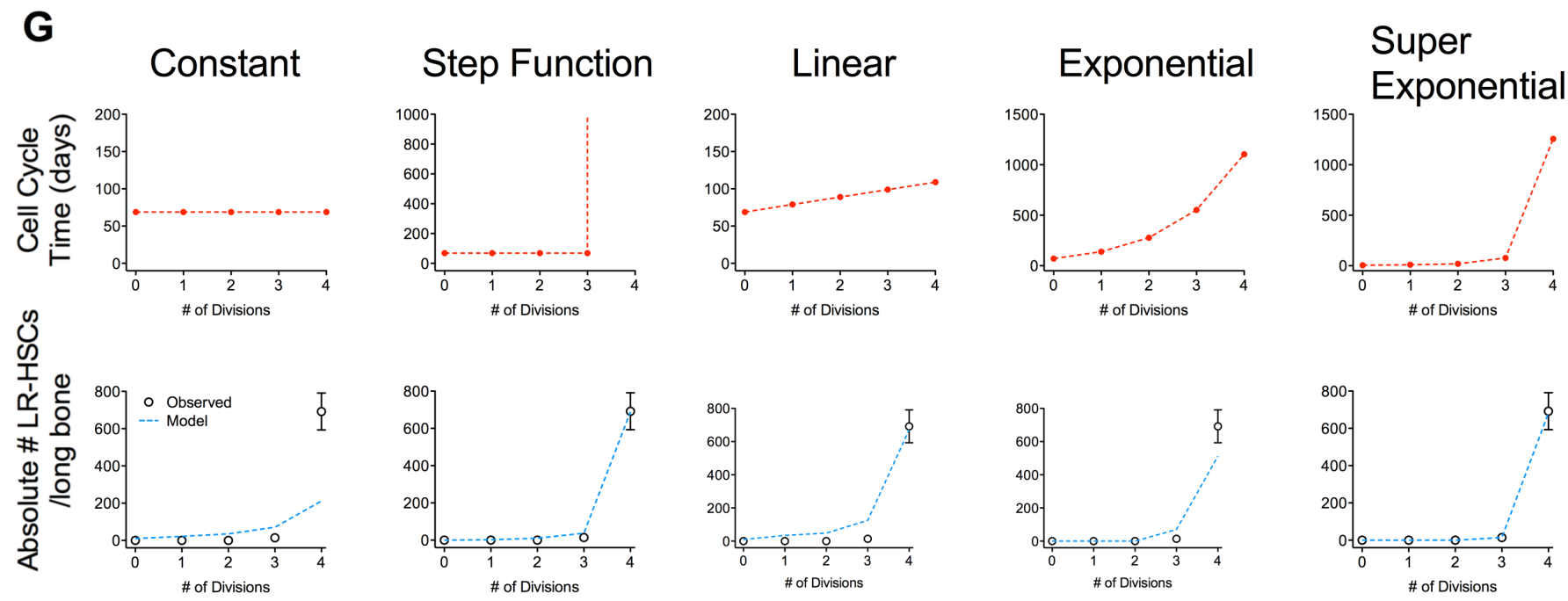

H
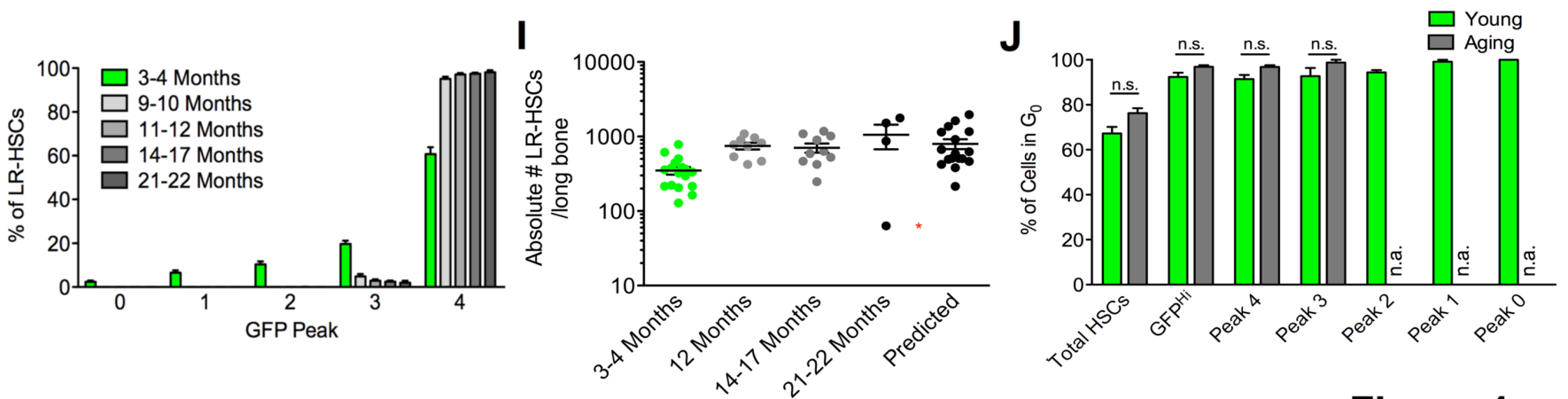

Figure 4 


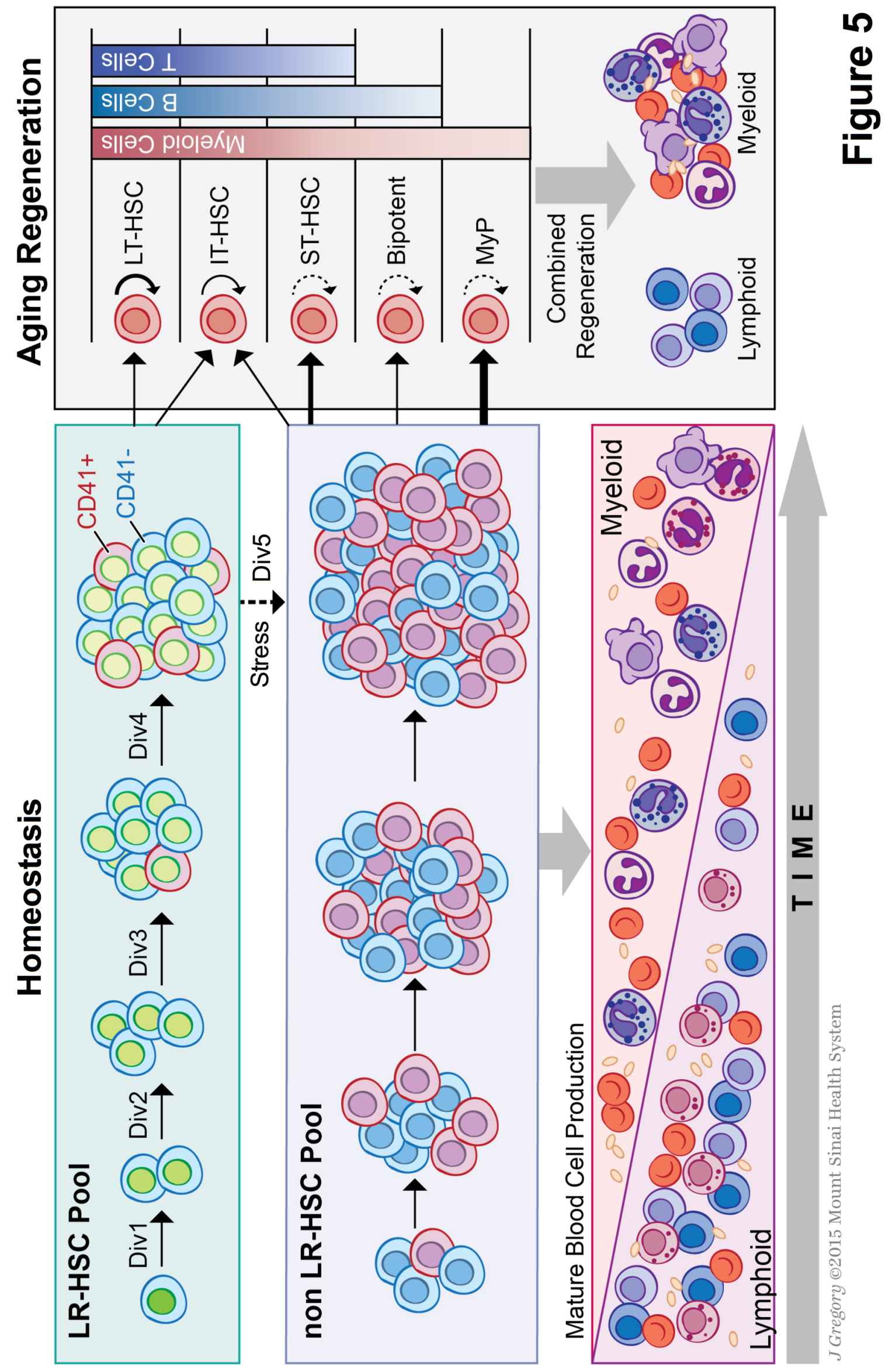


A LSKCD48-Flk2-CD150+ Gated
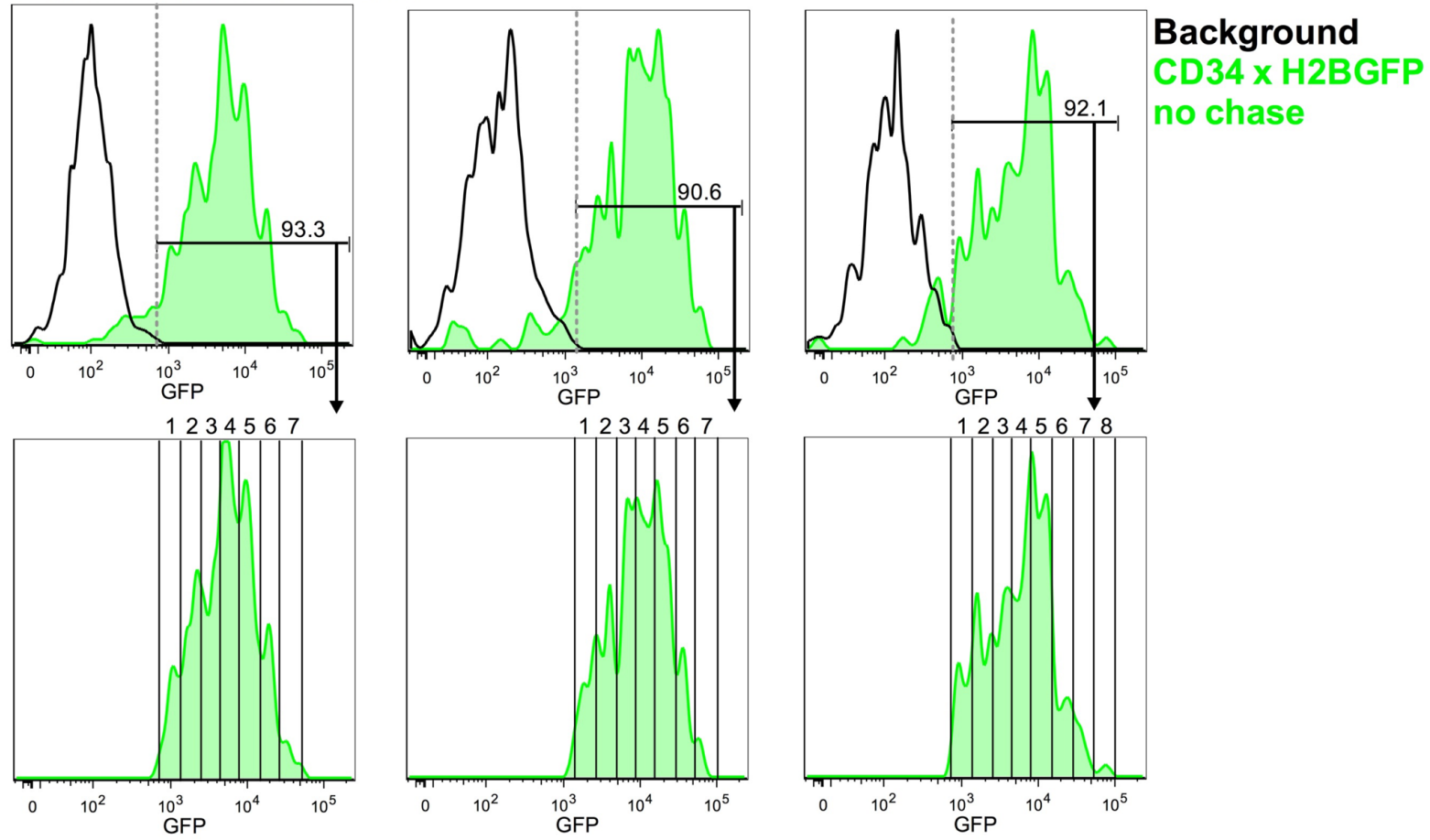

no chase

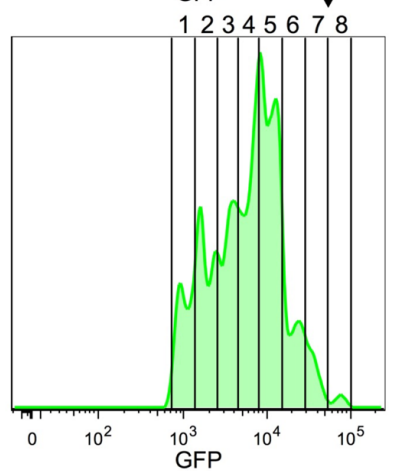

B

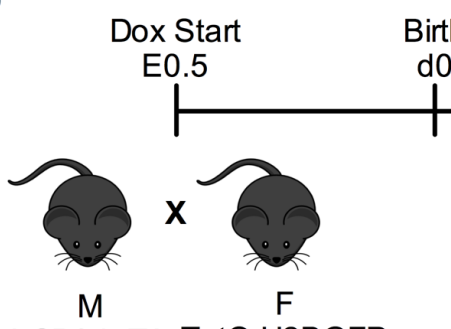

hCD34-tTA TetO-H2BGFP

C LSKCD48-CD150+ gated

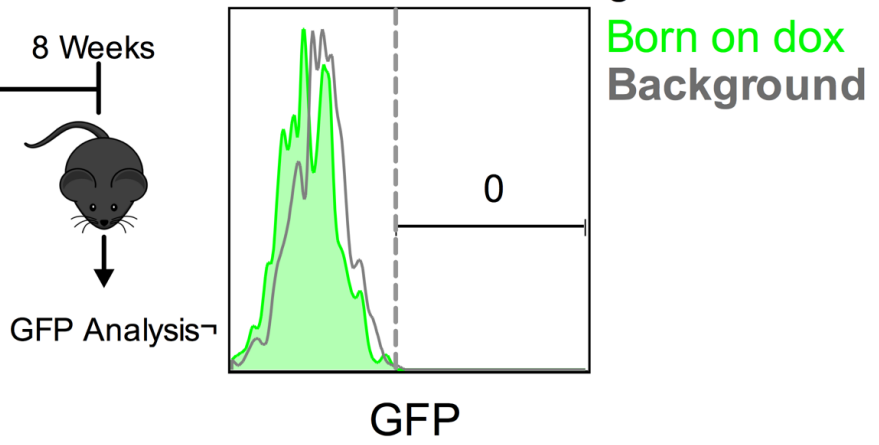

D

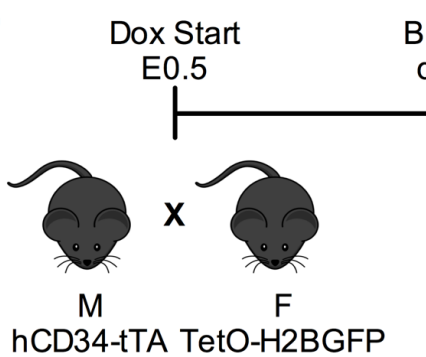
Bith do

E LSKCD48-CD150+ gated

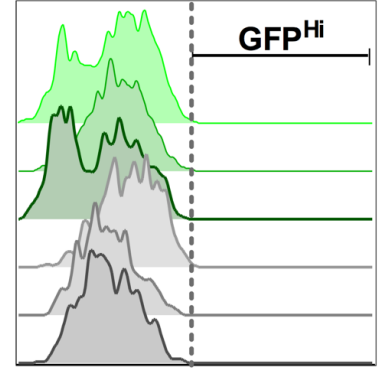

\section{Born on dox} Background

F

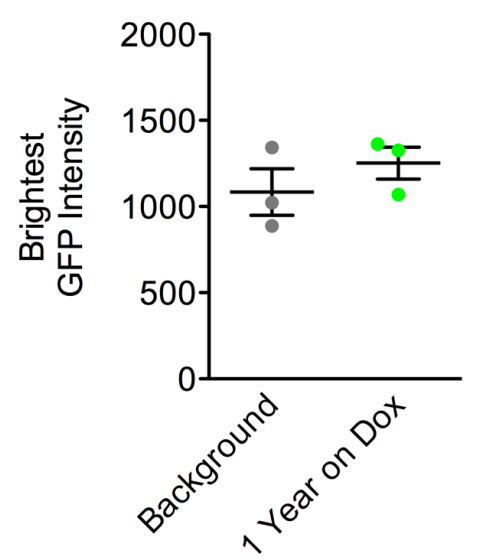

Figure S1 
A

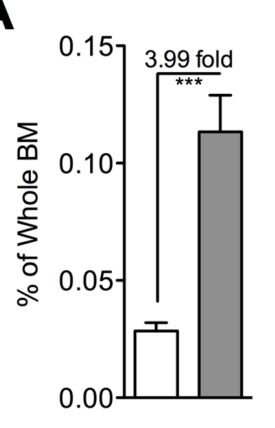

C

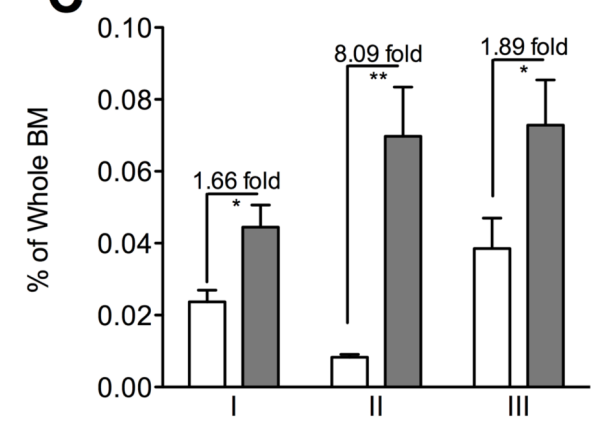

B

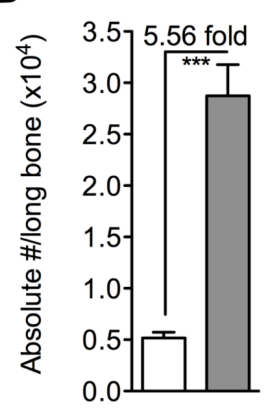

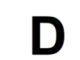

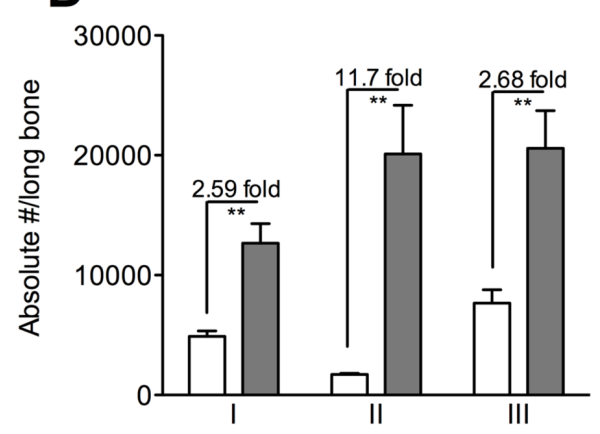

E

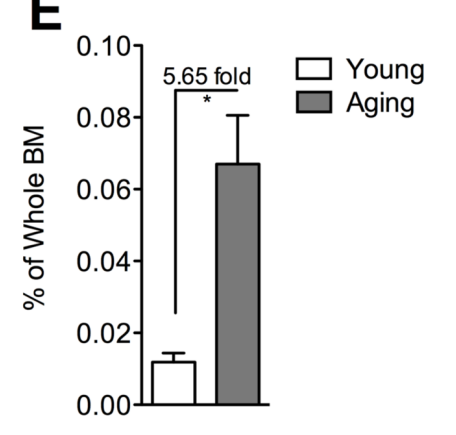

F

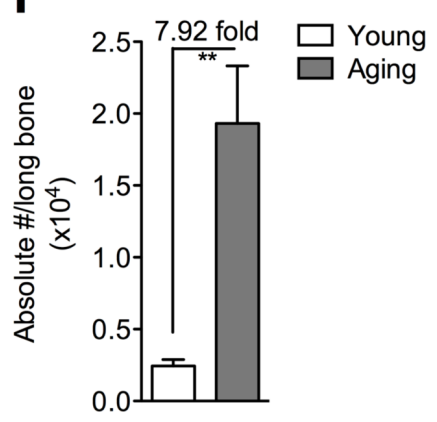

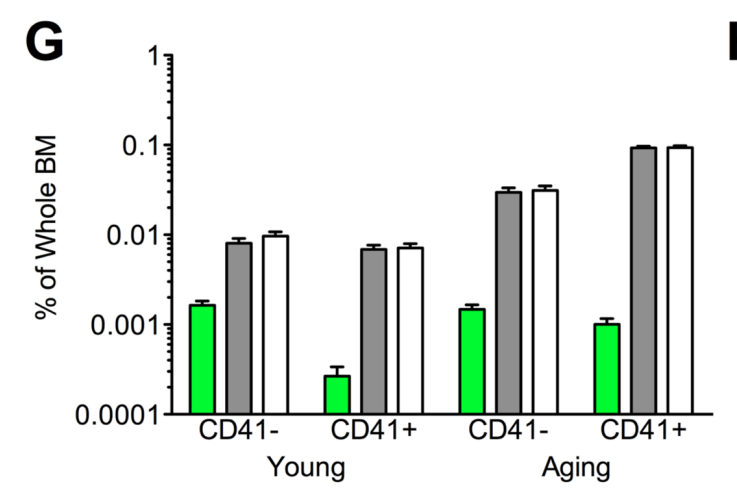



I

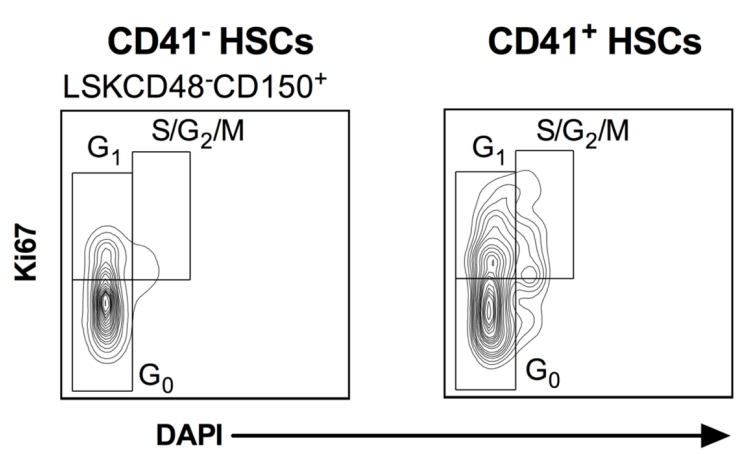

J
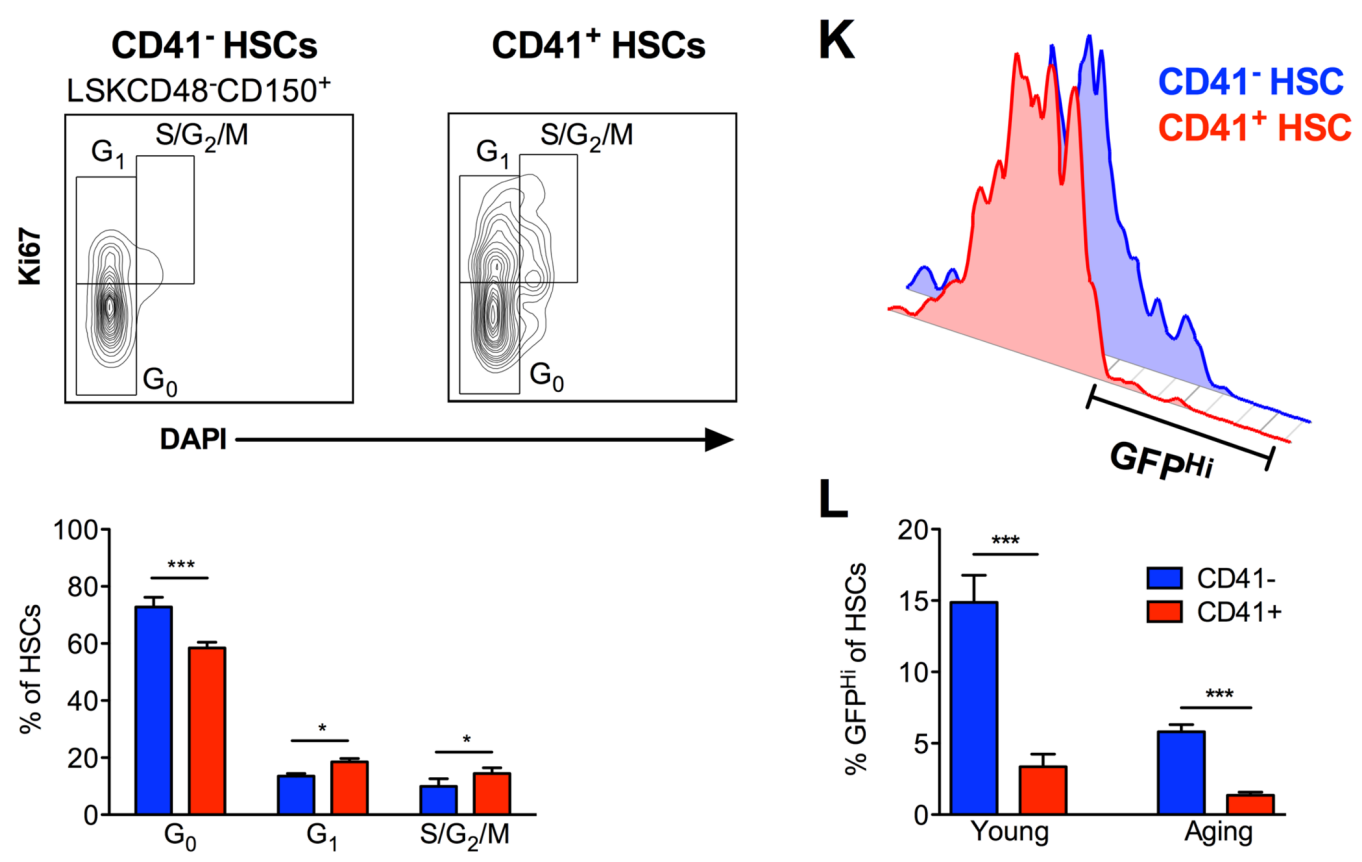

Figure S2 Related to Figure 2 


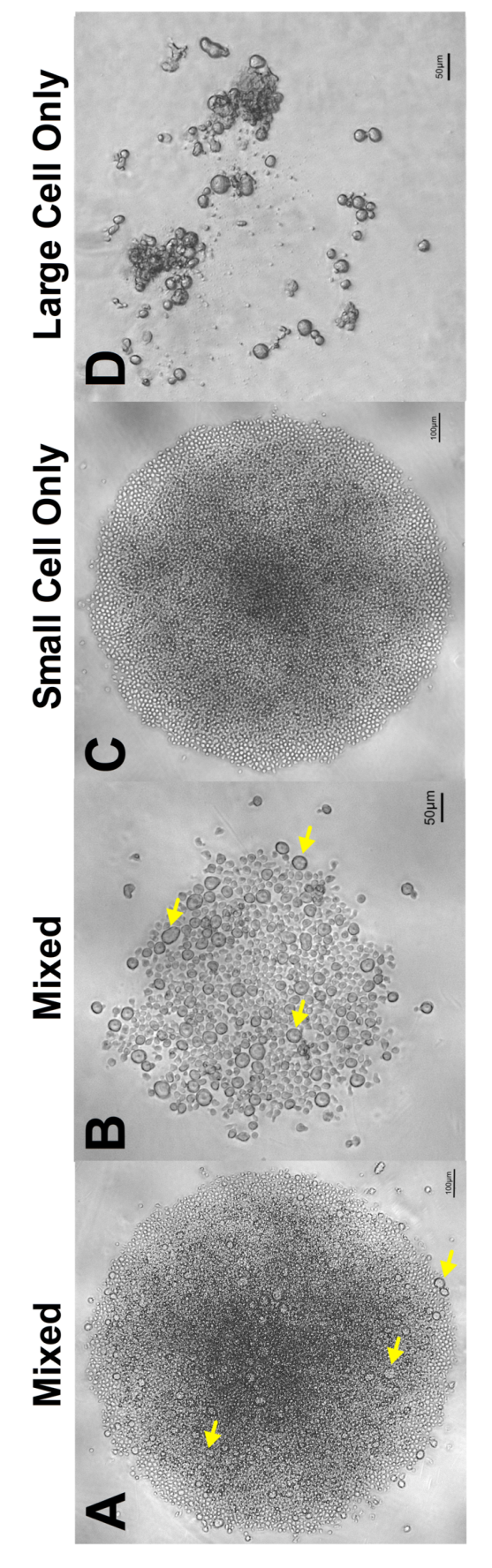

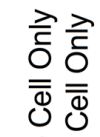

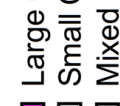

प००

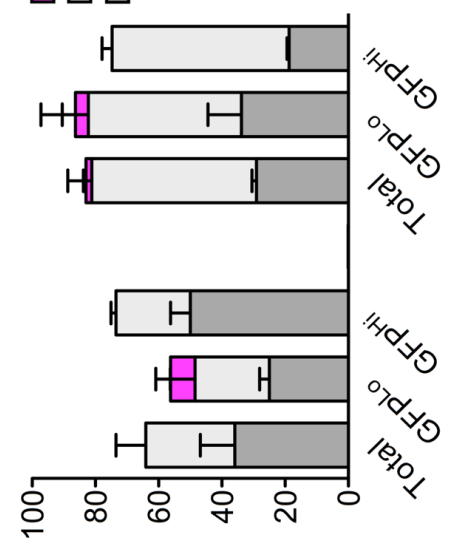



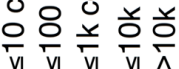

प्र००

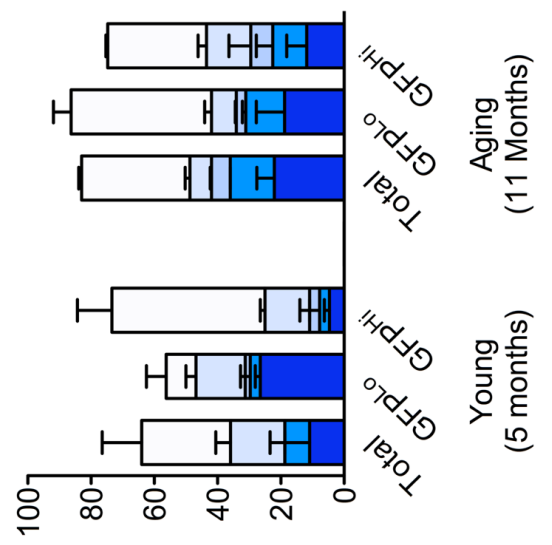

sə!uoloว pəunoঊ feut

s||әว ә|бu!S to \%

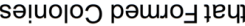

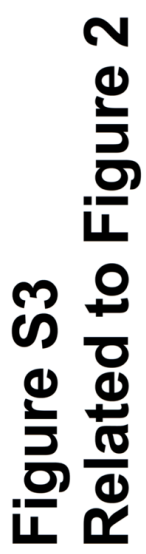

0

I

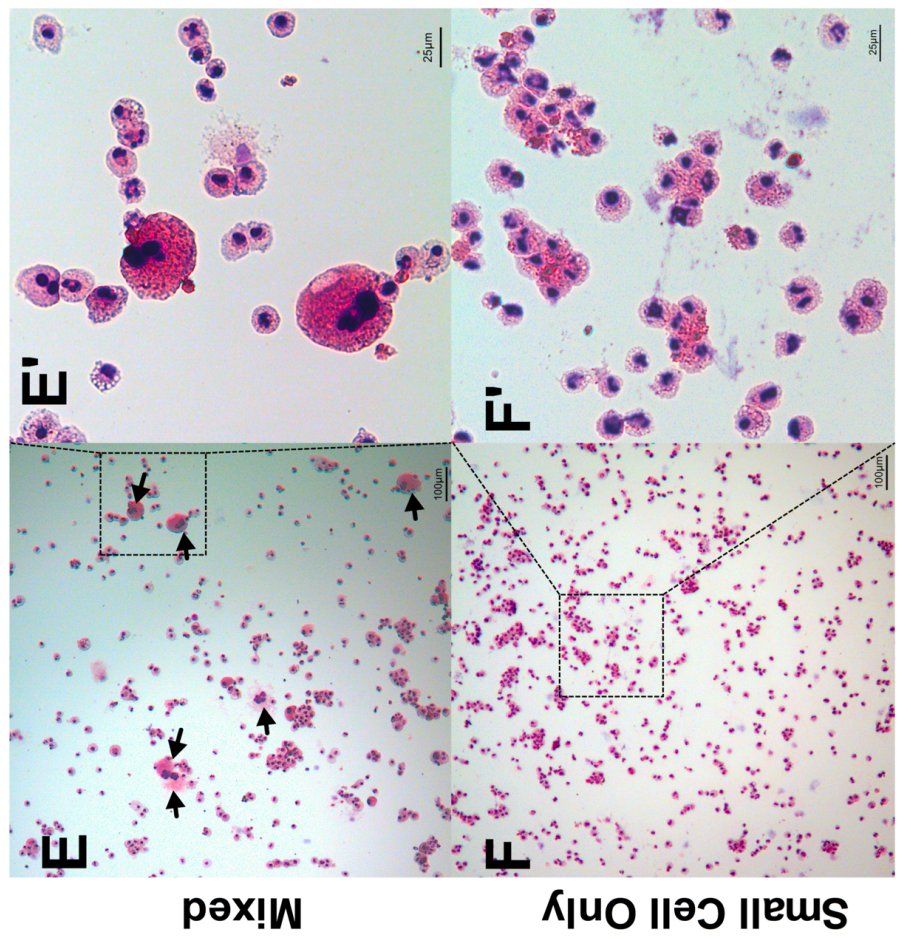



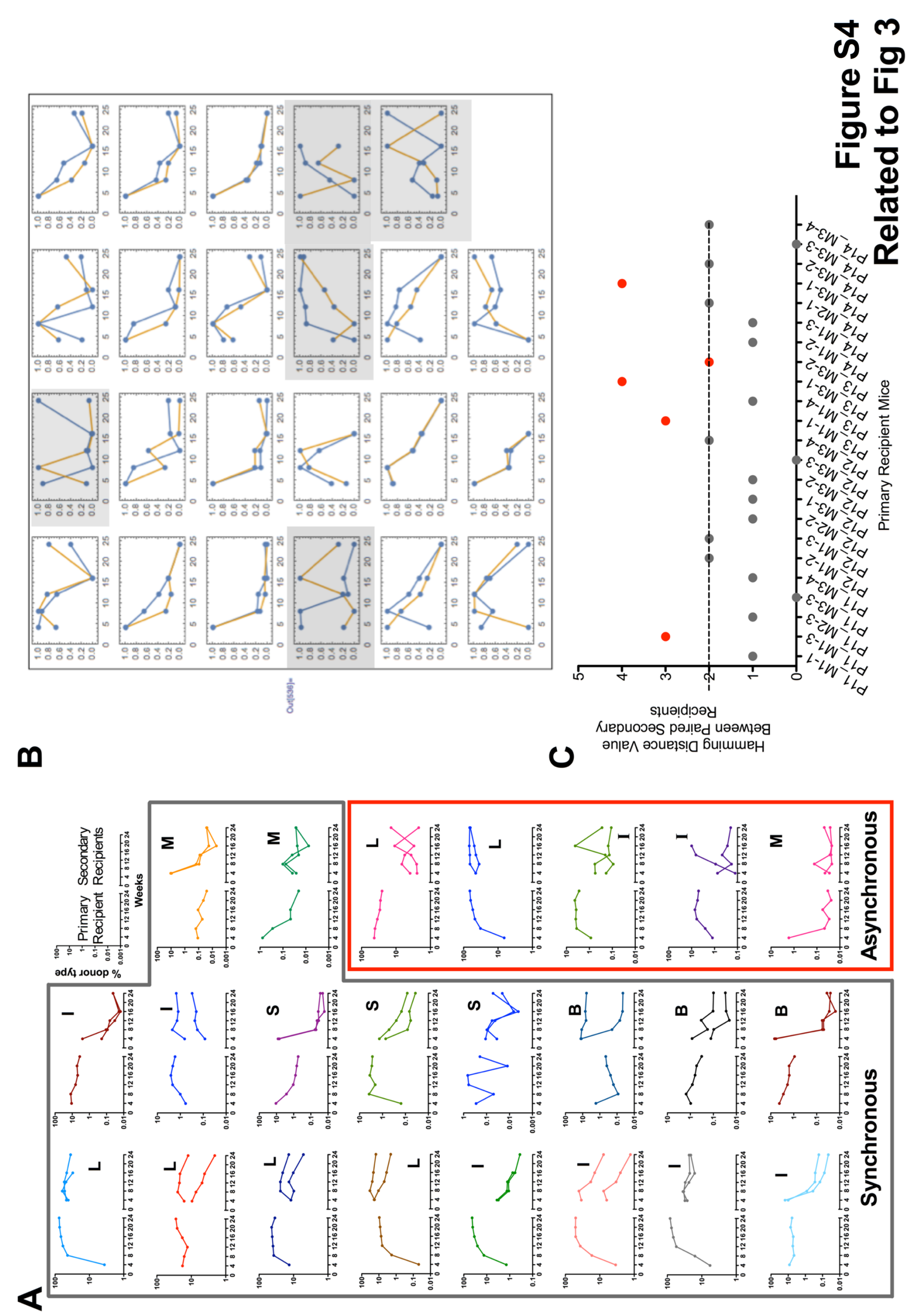


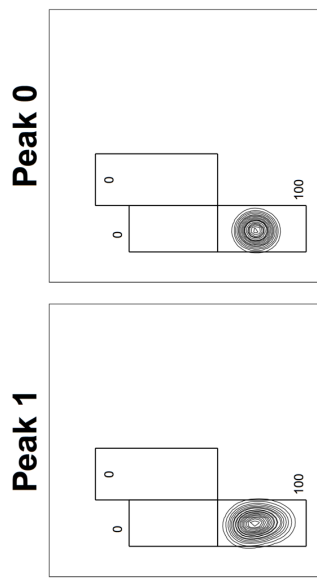

ণ্்
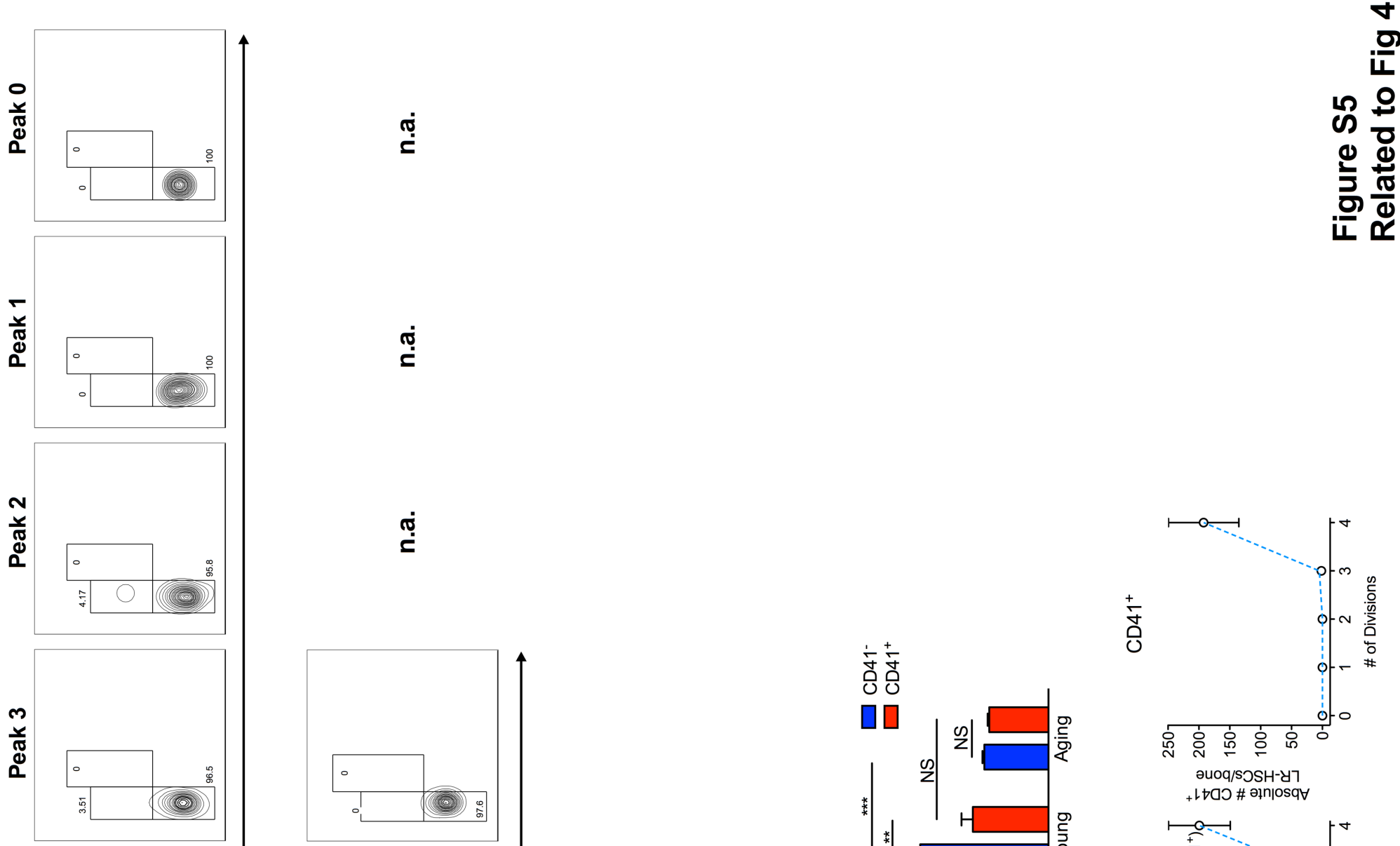

客竞

ֻே்

ণั่
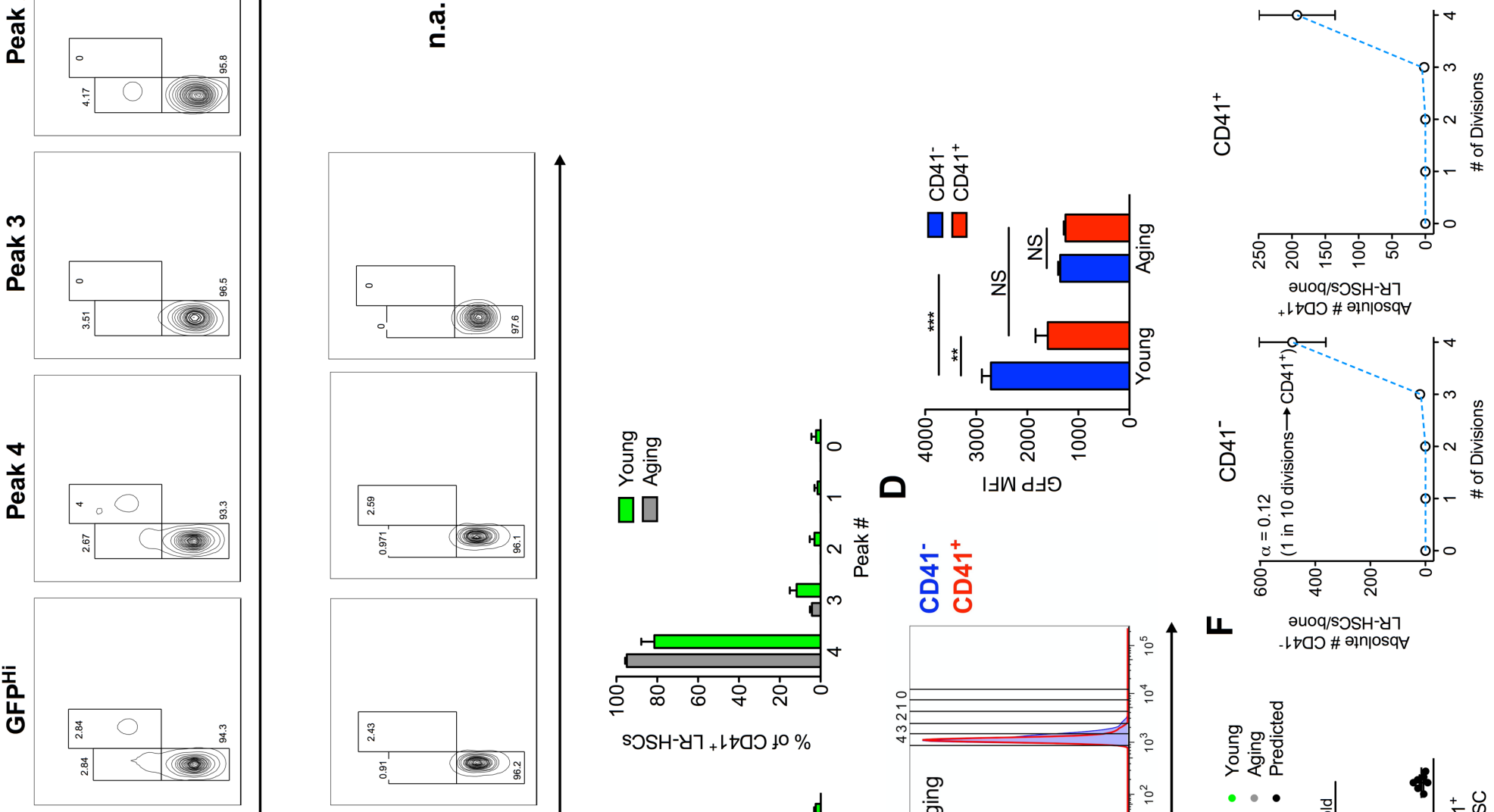

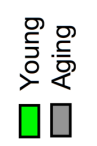

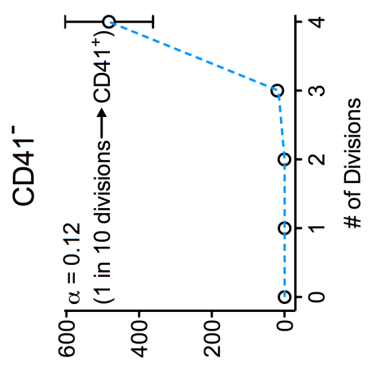

守 守
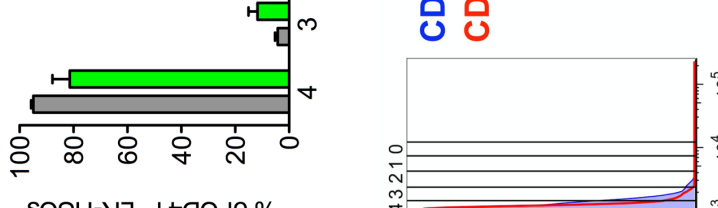

L

әuoq/soSH-บ7 - เ DO० \# әॄn|Osq

sวSH-yר + เtaว to \%
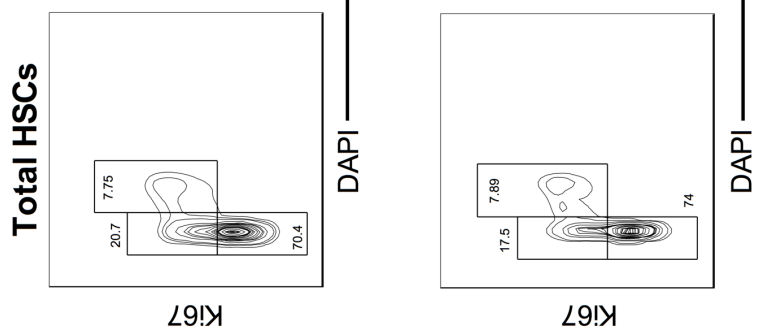

6u!n

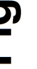

$\frac{2}{8}$

$\stackrel{\Phi}{\frac{\Phi}{\sigma}}$

番

$\varangle$

bunoג 